\title{
The Evolutionary Stability of Optimism, Pessimism, and Complete Ignorance*
}

\author{
Burkhard C. Schipper ${ }^{\dagger}$
}

December 9, 2020

\begin{abstract}
We seek an evolutionary explanation for why in some situations humans maintain either optimistic or pessimistic attitudes towards uncertainty and are ignorant to relevant aspects of their environment. Players in strategic games face Knightian uncertainty about opponents' actions and maximize individually their Choquet expected utility with respect to neo-additive capacities (Chateauneuf, Eichberger, and Grant, 2007) allowing for both an optimistic or pessimistic attitude towards uncertainty as well as ignorance to strategic dependencies. An optimist (resp. pessimist) overweighs good (resp. bad) outcomes. A complete ignorant never reacts to opponents' changes of actions. We focus on sub- and supermodular aggregative games and provide monotone comparative statics w.r.t. optimism/pessimism. With qualifications we show that in finite populations optimistic (resp. pessimistic) complete ignorance is evolutionary stable and yields a strategic advantage in submodular (resp. supermodular) games with aggregate externalities. Moreover, this evolutionary stable preference leads to Walrasian behavior in these classes of games.
\end{abstract}

Keywords: ambiguity, Knightian uncertainty, Choquet expected utility, neo-additive capacity, Hurwicz criterion, Minimax, supermodularity, aggregative games, monotone comparative statics, playing the field, evolution of preferences.

JEL-Classifications: C72, C73, D01, D43, D81, L13.

\footnotetext{
${ }^{*}$ I thank Adam Dominiak, Jürgen Eichberger, Burkhard Hehenkamp, Aviad Heifetz, Martin K. Jensen, David Kelsey, Jörg Oechssler, and seminar participants in Bonn and the 2005 Econometric Society World Congress in London for helpful discussions. Special thanks to Jürgen Eichberger, who together with David Kelsey introduced me to games with ambiguity, and with whom I had the fortune to collaborate on some applications of Choquet expected utility to games. The current paper is directly related to several of Jürgen's work, incl. Chateauneuf, Eichberger, and Grant (2007), Eichberger and Kelsey (2000, 2002, 2014), Eichberger, Kelsey, and Schipper (2008, 2009), and Eichberger and Guerdjikova (2018). Moreover, I thank the guest editor, Ani Guerdjikova, and two anonymous reviewers for helpful comments. Financial support by the DFG through SFB/TR 15 is gratefully acknowledged.

${ }^{\dagger}$ Department of Economics, University of California, Davis, Email: bcschipper@ucdavis.edu
} 


\section{Introduction}

The motivation for this work is twofold: First, we seek an evolutionary foundation for why humans maintain in some situations an optimistic or pessimistic attitude towards uncertainty and are ignorant to strategic aspects. Second, at a more theoretical level, we want to study how we can restrict by evolutionary arguments degrees of freedom in models with Knightian uncertainty, ambiguity, or imprecise beliefs. In particular, we want to endogeneize a player's attitude towards Knightian uncertainty as well as the amount of Knightian uncertainty over opponents' actions in strategic games by asking which of those parameters would maximize material payoffs (or fitness) and thus prosper and thrive.

In the literature on social psychology, there is evidence for both optimistic and pessimistic attitudes in the face of uncertainty and their relation to "success". For example, Seligman and Schulman (1986) found that more optimistic health insurance agents sold more policies during the first year of employment and were less likely to quit. Cooper, Dunkelberg, and Woo (1988), using interviews, found that self-assessed chances of new entrepreneurs' success are uncorrelated with education, prior experience, and start-up capital, and are overly optimistic. Taylor and Brown (1988) found that mentally healthy individuals maintain some unrealistic optimism whereas depressed individuals have more accurate perceptions. Studies on individual decision making show that the majority of subjects shy away from uncertain prospects like in the Ellsberg's paradox (for a survey see for example Camerer and Weber, 1992). To summarize, there is evidence on optimism in psychology and evidence on pessimism in the literature on individual decision making. This may suggest that both types of belief biases are present in the majority of the population and are not stable across situations. An individual may hold optimistic beliefs in some situations but pessimistic beliefs in some other situations. In this article we seek an evolutionary explanation and show that these biases may depend on the strategic situation.

We model Knightian uncertainty, ambiguity or imprecise beliefs by Choquet expected utility theory (CEU) with non-extreme-outcome-additive (neo-additive) capacities introduced by Chateauneuf, Eichberger, and Grant (2007). Knightian uncertainty or ambiguity refers to situations where probabilities may be unknown or imperfectly known as opposed to situations under risk where probabilities are known, a distinction made by Knight (1921). CEU with respect to neo-additive capacities by Chateauneuf, Eichberger, and Grant (2007) includes as special cases Subjective expected utility (SEU) as well as various preferences for decision making under complete ignorance such as Minimax (Wald, 1951), Maximax, and Hurwicz preferences (Hurwicz, 1951, Arrow and Hurwicz, 1972). More importantly, because of its parameterized form, it lends itself well to applications.

Since CEU with neo-additive capacities by Chateauneuf, Eichberger, and Grant (2007) is a generalization of conventional Subjective expected utility theory (SEU), it has more degrees of freedom like the degrees of ignorance and the degrees of optimism and pessimism defined in

the next section. It is natural to ask how to select among the degrees of optimism/pessimism and ignorance. A possible answer could be provided in an indirect evolutionary framework: If evolution (including social learning processes and market selection) chooses preferences parameterized by those degrees of freedom, which one would it choose? To study such questions, we make use of the literature on Choquet expected utility in strategic games, in which players face Knigthian uncertainty about opponents' actions (see for instance Dow and Werlang, 1994, Eich- 
berger and Kelsey, 2000, 2002, 2014, Marinacci, 2000, Eichberger, Kelsey, and Schipper, 2009). ${ }^{1}$ While it is intuitive that players face Knightian uncertainty in an unfamiliar one-shot situation, we show that even after a very large number of repeated interactions implicitly assumed to be behind evolution, players may be still have biased beliefs and be completely ignorant.

We focus on submodular games and supermodular games (see Topkis, 1998) both with aggregation and positive or negative externalities. These classes include many games prominent in economics and social sciences in general. Many games in economics involve ordered action sets like prices, quantities, qualities, contribution levels, appropriation levels etc. Often there is a natural aggregate of all players actions like total market quantity, total contribution or appropriation etc. ${ }^{2}$ Moreover, many games with ordered action space have either some version of strategic substitutes or strategic complements (Bulow, Geanakoplos and Klemperer, 1985) or can be brought into a framework of supermodular or submodular games. ${ }^{3}$ We show that sub- and supermodularity is preserved when extending these games to Choquet expected utility with neo-additive capacities. Moreover, Choquet expected utility with neo-additive capacities features distinct parameters such as the degree of ignorance and degree of optimism. As we will show using results from Topkis (1998), these parameters lend themselves well to monotone comparative statics.

The monotone comparative statics of equilibrium under Knightian uncertainty with respect to the degree optimism/pessimism is just a first stepping stone in studying the restriction of parameters of CEU with neo-additive capacities by evolutionary arguments. It allows us to learn about changes in behavior when mutants with different preference parameters enter the population of players. We use the indirect evolutionary approach (Güth and Yaari, 1992, Güth, 1995, Heifetz, Shannon, and Spiegel, 2007a, b; see Alger and Weibull, 2019, for a recent survey). In this literature, players drawn randomly from a large population are matched to play a game and behave rationally with respect to their preferences but their survival is judged by their material payoff. In our contexts, preferences are given by CEU w.r.t. neo-additive capacities while material payoffs are payoffs in games. Instead of working with a large population, we employ Schaffer's $(1988,1989)$ notion of evolutionary stability for finite populations because we believe that in many situations of economic relevance, players "play the field", i.e., all players in a finite set of players play a game. A strategy is finite population evolutionary stable if it maximizes relative (material) payoffs among all players. The technical motivation is that finite population evolutionary strategies are relatively easy to work with in sub- and supermodular aggregative games (Schipper, 2003, Alós-Ferrer and Ania, 2005). With this apparatus, we can then ask which parameter configurations of CEU with neo-additive capacities yield actions in equilibrium under Knightian uncertainty that maximize relative material payoffs. These

\footnotetext{
${ }^{1}$ There is a growing literature on ambiguity in strategic games. Beside the aforementioned papers, it includes Klibanoff (1996), Lo (1996, 1999), Epstein (1997), Groes et al. (1998), Haller (2000), Ryan (2002), Bade (2011), Jungbauer and Ritzberger (2011), Riedel and Sass (2014), Battigalli et al. (2015, 2016), Dominiak and Schipper (2019) etc.

${ }^{2}$ For games with aggregation see Schipper (2003), Alós-Ferrer and Ania (2005), Cornes and Hartley (2005), Jensen (2005), and Acemoglu and Jensen (2013).

${ }^{3}$ Examples include Cournot oligopoly (Amir, 1996, Vives, 2000), some Bertrand oligopoly (Vives, 2000), common pool resource dilemma (Walker, Gardner, and Ostrom, 1990), some rent seeking games (Hehenkamp, Leininger and Possajennikov, 2004), some bargaining games, some public goods games, some co-ordination games (e.g. Cooper, 1999), arms race and search problems (Milgrom and Roberts, 1990).
} 
parameter configurations are the finite population evolutionary stable preferences.

To build an intuition for our results, consider for example a version of a Nash bargaining game. Let there be two players who simultaneously demand a share of a fixed pie. If the demands sum up to less than $100 \%$ of the pie, then the pie is shared according to the demands. Otherwise, players receive nothing. This game has strategic substitutes because the marginal benefit from a player's larger demand is decreasing in the demand of the opponent. Let each player face Knightian uncertainty about the opponents' demand. Suppose that a player is a pessimist. Then she overweighs large demands by the opponent. The more pessimistic the belief, the lower is the best response demand because the player fears the incompatibility of demands, which would result in a zero payoff. Could such a pessimistic belief be evolutionary stable? Suppose the opponent (the "mutant") is not as pessimistic, then his best response is a larger demand. If demands add up to less than 100\%, this opponent is strictly better off than the pessimist, otherwise both get nothing and he is not worse off. Thus pessimism can not be evolutionary stable in this two-player game. Is optimism evolutionary stable? Suppose the opponent is an extreme optimist in the sense that he believes that the opponent will demand zero, then the best response is to demand $100 \%$. If the opponent does indeed demand zero, the extreme optimist is strictly better off. Otherwise, if the opponent does demand some strict positive share, then both receive nothing and the extreme optimist is not worse off. Thus there is no preference with an attitude towards Knightian uncertainty that would successfully invade a set of extremely optimistic players because an optimist can not be made worse off relative to other player.

The example of the Nash bargaining game is also an example for the evolutionary stability of complete ignorance. A completely ignorant player behaves as if she is alone and there is no opponent. We will show that evolutionary stability of (to some degree) optimistic preferences with complete ignorance holds with qualifications not only for the Nash bargaining game but for an entire class of games characterized by a general notion of strategic substitutes, submodular aggregative games. Similarly, we show that preferences reflecting extreme pessimism and complete ignorance are finite population evolutionary stable in games with some general notion of strategic complements, supermodular aggregative games.

The evolutionary stability of ignorance is somewhat disappointing given that we put in considerable effort in modelling games with a rich class of preferences. At a second glance it is not surprising though. Ignorance yields commitment power. As the example of the Nash demand game illustrates, there are important classes of games where commitment to an aggressive action maximizes relative material payoffs. We should point out though that we present just sufficient conditions. Moreover, we demonstrate with examples that complete ignorance is not necessary for finite population evolutionary stability. Yet, in order to really take advantage of the rich class of CEU preferences in strategic settings and go beyond complete ignorance, we believe it will be necessary to go beyond the important classes of games that we consider. The evolutionary stability of optimism versus pessimism depends on the classes of games we study. In submodular aggregative, a bolder aggressive action maximizes relative material payoffs and hence optimism becomes evolutionary stable. This insight goes back essentially to von Stackelberg (1934). In contrast, in our class of supermodular aggregative games, a timid action maximizes relative material payoffs and hence pessimism is evolutionary stable, an insight that becomes immediately intuitive when considering for instance minimum effort coordination games. In both classes of games, the evolutionary stable preference leads to Walrasian behavior 
meaning that players behave as if they maximize material payoffs taking the aggregate of all players actions as given.

How to interpret this evolutionary approach? While we trust that the formal results will become clear in the following sections, it is less immediate how to understand the notion of evolution. In an economic context, evolution may not necessarily be understood in a biological sense. Rather we may be primarily concerned with the question as to what extent a trait (here either optimism or pessimism and ignorance) is relatively more successful than (or yields a strategic advantage over) other traits (i.e., other attitudes towards uncertainty). These successful traits may then be spread through social imitation (Vega-Redondo, 1997, Schipper, 2009, Duersch, Oechssler, and Schipper, 2012). Beyond "social learning", there is some empirical evidence on a positive correlation between economic success and reproductive success from both today's developing countries (Hull and Hull, 1977, Mace, 1996, Mulder, 1987) and some European countries prior to the industrial revolution (Boone, 1986, Hughes, 1986). So even a biological interpretation of our notion of evolution may not be too far-fetched after all.

While we are not aware of any prior results seeking to restrict parameters of Choquet expected utility by evolutionary arguments in games, the associate editor kindly made us aware that Eichberger and Guerdjikova (2018) study the evolution of optimism and pessimism in a financial market using a case-based $\alpha$-MEU model and show that pessimists may dominate the market in the long-run. Our results on the monotone comparative are closely related to Eichberger, Kelsey, and Schipper (2009) and Eichberger and Kelsey (2014). We extend earlier work by Eichberger, Kelsey, and Schipper (2009) who study Choquet expected utility with neo-additive capacities in particular examples such as Cournot duopoly, Bertrand duopoly, strategic delegation, a "peace-making" game, and some two-player concave games with strategic complementarities. Eichberger and Kelsey (2014) model optimism and pessimism in games with Choquet expected utility featuring JP-capacities (Jaffray and Philippe, 1997), which are more general than neo-additive capacities. They also make use of Topkis' (1998) results on monotone comparative statics in supermodular games but restrict to finite one-dimensional action sets. They do not consider submodular games though.

The article has two main sections: The next section provides a comprehensive step-by-step exposition of the theory of sub- and supermodular aggregative games with Choquet expected utility with neo-additive capacities including monotone comparative statics. Section 3 focuses on evolutionary stable preferences. We conclude with a discussion in Section 4. Proofs are relegated to the appendix.

\section{Super- and Submodular Aggregative Games with Ambiguity}

In this section, we present the theory of super- and submodular aggregative games with negative or positive externalities under Choquet expected utility w.r.t. neo-additive capacities. This is the class of games for which we study finite-population evolutionary stable preferences in Section 3 . 


\subsection{Preliminaries}

We first introduce some order-theoretic notions with which we define our class of games. A partially ordered set $\langle X, \unrhd\rangle$ is a set $X$ with a binary relation $\unrhd$ that is reflexive, antisymmetric, and transitive. The dual of a set $X$ with a partial order $\unrhd$ is the same set $X$ with a partial order $\unrhd^{\prime}$ such that for $x^{\prime}, x^{\prime \prime} \in X, x^{\prime} \unrhd^{\prime} x^{\prime \prime}$ if and only if $x^{\prime \prime} \unrhd x^{\prime}$. A chain is a partially ordered set that does not contain an incomparable pair of elements, i.e., a totally or completely ordered set. A lattice $\langle X, \unrhd\rangle$ is a partially ordered set in which each pair of elements $x, y \in X$ has a least upper bound (join) denoted by $x \vee y=\sup _{X}\{x, y\}$ and a greatest lower bound (meet) denoted by $x \wedge y=\inf _{X}\{x, y\}$ contained in this set. A lattice $\langle X, \unrhd\rangle$ is complete if for every nonempty $Y \subseteq X, \sup _{X} Y$ and $\inf _{X} Y$ exist in $X$. A sublattice $Y$ of a lattice $X$ is a subset $Y \subseteq X$ for which each pair of elements in $Y$ the join and meet is contained in $Y$. A sublattice $Y$ of a lattice $X$ is subcomplete if for each nonempty subset $Y^{\prime} \subseteq Y, \sup _{X}\left(Y^{\prime}\right)$ and $\inf _{X}\left(Y^{\prime}\right)$ exist and are contained in $Y$. The interval-topology on a lattice $X$ is the topology for which each closed set is either $X, \emptyset$, or of type $\{y \in X \mid x \unrhd y, y \unrhd z\}$. A lattice is complete if and only if it is compact in its interval-topology (see Topkis, 1998, pp. 29). We assume that all lattices are endowed with a topology finer than the interval-topology, and that all products of topological spaces are endowed with the product topology. ${ }^{4}$ For a lattice $\langle X, \unrhd\rangle$ with $A, B \subseteq X, B$ is higher than $A$ if $a \in A, b \in B$ implies that $a \vee b \in B$ and $a \wedge b \in A$ (strong set order). We abuse notation and write $B \unrhd A$. A function (correspondence) $f$ from a partially ordered set $X$ to a partially ordered set $Y$ is increasing (decreasing) if $x^{\prime \prime} \unrhd x^{\prime}$ in $X$ implies $f\left(x^{\prime \prime}\right) \unrhd(\unlhd) f\left(x^{\prime}\right)$. It is strictly increasing (decreasing) if we replace " $\unrhd(\unlhd)$ " with its non-reflexive part " $\triangleright(\triangleleft)$ " in the previous sentence.

Definition 1 (Supermodular/Submodular) A real valued function $f: X \longrightarrow \mathbb{R}$ on a lattice $X$ is supermodular in $x$ on $X$ if for all $x, y \in X$,

$$
f(x)+f(y) \leq f(x \vee y)+f(x \wedge y) .
$$

A real valued function on a lattice is submodular if $-f$ is supermodular. A function that is both supermodular and submodular is called a valuation.

Definition 2 (Increasing / Decreasing Differences) A real valued function $f$ on a partially ordered set $X \times T$ has increasing (decreasing) differences in $(x, t)$ on $X \times T$ if for all $x^{\prime \prime} \triangleright x^{\prime}$ and $t^{\prime \prime} \triangleright t^{\prime}$,

$$
f\left(x^{\prime \prime}, t^{\prime}\right)-f\left(x^{\prime}, t^{\prime}\right) \leq(\geq) f\left(x^{\prime \prime}, t^{\prime \prime}\right)-f\left(x^{\prime}, t^{\prime \prime}\right) .
$$

If inequality (2) holds strictly, then $f$ has strictly increasing (decreasing) differences.

As a special case, functions defined on a finite product of chains that have increasing differences on this product are also supermodular on this product (Topkis, 1998, Corollary 2.6.1.). A familiar characterization of increasing differences in many economic problems is as follows: If $f: \mathbb{R}^{n} \longrightarrow \mathbb{R}$ is twice-continuously differentiable, then $f$ has (strictly) increasing differences on $\mathbb{R}^{n}$ if and only if $\frac{\partial^{2} f(x)}{\partial x_{i} \partial x_{j}} \geq(>) 0$ for all $i \neq j$ and $x$. An analogous result holds for (strictly) decreasing differences.

\footnotetext{
${ }^{4}$ This is important later for existence of equilibrium under Knightian uncertainty.
} 
The importance of the properties stem from the interpretation of $f$ as a payoff function. For example, decreasing differences of payoffs in a player's own action and some aggregate of all players' actions is read as: Consider a symmetric $n$-player game. Let player 1 take a larger action than player 2. Then the difference between their payoffs is decreasing in the aggregate of all players' actions. This will be become clear in the next subsections.

\subsection{Strategic Games with Ordered Actions}

Let $N$ be the finite set of players $i=1, \ldots, n$. Each player's set of actions is a sublattice $A_{i}$ of a lattice $X$. We write $\mathbf{A}=\times_{i \in N} A_{i}$, with a typical element being $\mathbf{a} \in \mathbf{A}$. Player $i$ 's payoff function is $\pi_{i}: \mathbf{A} \longrightarrow \mathbb{R}$. We denote by $A_{-i}=\times_{j \in N \backslash\{i\}} A_{j}$. A typical element of $A_{-i}$ is $a_{-i}$. $G=\left\langle N,\left(A_{i}\right),\left(\pi_{i}\right)\right\rangle$ denotes a strategic game (with lattice action space). A Nash equilibrium in pure actions of the strategic game $G$ is an action profile $\mathbf{a}^{*} \in \mathbf{A}$ such that for all $i \in N$,

$$
\pi_{i}\left(a_{i}^{*}, a_{-i}^{*}\right) \geq \pi_{i}\left(a_{i}, a_{-i}^{*}\right) \text {, for all } a_{i} \in A_{i} .
$$

Let $\mathcal{E}(G) \subseteq \mathbf{A}$ denote the set of pure Nash equilibria of the game $G$.

A strategic game $G$ is (strictly) supermodular if for each player $i \in N$ the payoff function $\pi_{i}$ is supermodular in $a_{i}$ on $A_{i}$ for each $a_{-i} \in A_{-i}$ and has (strictly) increasing differences in $\left(a_{i}, a_{-i}\right)$ on $\mathbf{A}$. A strategic game $G$ is (strictly) submodular if for each player $i \in N$ the payoff function $\pi_{i}$ is (strictly) supermodular in $a_{i}$ on $A_{i}$ for each $a_{-i} \in A_{-i}$ and has (strictly) decreasing differences in $\left(a_{i}, a_{-i}\right)$ on $\mathbf{A}$.

A strategic game $G$ has positive (negative, resp.) externalities if for each player $i \in N$ the payoff $\pi_{i}\left(a_{i}, a_{-i}\right)$ is increasing (decreasing, resp.) in $a_{-i}$ on $A_{-i}$ for each $a_{i} \in A_{i}$. A strategic game $G$ has strictly positive (negative, resp.) externalities if the strict versions hold.

Externalities have implications for the best and worst actions by opponents. The proof of the following observations is contained in the appendix.

Lemma 1 Assume that for each player $i \in N$, the set of actions $A_{i}$ is a subcomplete sublattice of a complete lattice $X$ and that $\pi_{i}$ is continuous in $a_{-i}$ on $A_{i}$ for every $a_{i} \in A_{i}$. If $G$ has positive externalities, then $\sup _{A_{-i}} A_{-i} \subseteq \arg \max _{a_{-i} \in A_{-i}} \pi_{i}\left(a_{i}, a_{-i}\right)$ and $\inf _{A_{-i}} A_{-i} \subseteq$ $\arg \min _{a_{-i} \in A_{-i}} \pi_{i}\left(a_{i}, a_{-i}\right)$ for all $a_{i} \in A_{i}$. If $G$ has negative externalities, then $\inf _{A_{-i}} A_{-i} \subseteq$ $\arg \max _{a_{-i} \in A_{-i}} \pi_{i}\left(a_{i}, a_{-i}\right)$ and $\sup _{A_{-i}} A_{-i} \subseteq \arg \min _{a_{-i} \in A_{-i}} \pi_{i}\left(a_{i}, a_{-i}\right)$ for all $a_{i} \in A_{i}$.

At this point, it may be useful to illustrate the concepts introduced so far with an example. The following example is not meant to appreciate the generality of our approach. Rather, it should help to reader to recognize the notions introduced so far in a simple familiar example.

Example 1 (Cournot duopoly with linear demand and convex cost) Consider two players, $i=1,2$, and symmetric payoff functions $\pi\left(a_{i}, a_{3-i}\right)=\left(100-a_{i}-a_{3-i}\right) a_{i}-\frac{1}{2} a_{i}^{2}$ with actions $a_{i}$ being in a suitable positive real-valued interval. This is a standard Cournot duopoly with linear demand and quadratic cost. Since each player $i$ 's set of actions is a real-valued interval, $\pi_{i}$ is trivially supermodular in $a_{i}$ for every $a_{3-i}$. It has strictly decreasing differences, $\frac{\partial^{2} \pi_{i}\left(a_{i}, a_{3-i}\right)}{\partial a_{i} \partial a_{3-i}}=-1$.

It has negative externalities, i.e., $\frac{\partial \pi_{i}\left(a_{i}, a_{3-i}\right)}{\partial a_{3-i}}=-a_{i}$ since $a_{i} \geq 0$. If $a_{i} \in A=[\underline{a}, \bar{a}]$ with $\underline{a}>0$ for $i=1,2$, then $\bar{a} \in \arg \min _{a_{3-i} \in A} \pi_{i}\left(a_{i}, a_{3-i}\right)$ and $\underline{a} \in \arg \max _{a_{3-i} \in A} \pi_{i}\left(a_{i}, a_{3-i}\right)$ for all $a_{i}$, 
illustrating Lemma 1.

For some of the results, we restrict attention to games in which players consider an aggregate of opponents' actions.

Definition 3 (Aggregative Game) A strategic game $G$ with ordered action space is aggregative if there exists an aggregator $\aleph: X \times X \longrightarrow X$ such that

(i) Idempotence: $\aleph^{1}\left(a_{i}\right):=a_{i}$ for all $a_{i} \in A_{i} \subseteq X$ and all $i \in N$;

(ii) Induction: $\aleph^{k}\left(a_{1}, \ldots, a_{k}\right)=\aleph\left(\aleph^{k-1}\left(a_{1}, \ldots, a_{k-1}\right), a_{k}\right)$, for $k=2, \ldots, n$;

(iii) Symmetry: $\aleph^{k}$ is symmetric for $k=1, \ldots, n$, i.e., $\aleph^{k}\left(a_{1}, \ldots, a_{k}\right)=\aleph^{k}\left(a_{f(1)}, \ldots, a_{f(k)}\right)$ for all bijections $f:\{1, \ldots, k\} \longrightarrow\{1, \ldots, k\}$, for $k=1, \ldots, n$

(iv) Order-preservation: $\aleph^{k}$ is order-preserving for $k=1, \ldots, n$, i.e., $\left(a_{1}, \ldots, a_{k}\right) \unlhd\left(a_{1}^{\prime}, \ldots, a_{k}^{\prime}\right)$ implies $\aleph^{k}\left(a_{1}, \ldots, a_{k}\right) \unlhd \aleph^{k}\left(a_{1}^{\prime}, \ldots, a_{k}^{\prime}\right)$, for $k=1, \ldots, n$;

and the payoff function $\pi_{i}$ is defined on $A_{i} \times X$ for all players $i \in N$.

As mentioned in the introduction, many games with ordered action sets in the literature have some aggregation property. A special example of an aggregative game would be the Cournot oligopoly where quantities are usually summed up to the market quantity. Definition 3 is similar to Alós-Ferrer and Ania (2005). Some versions of aggregative games are also considered by Corchón (1994), Dubey, Haimanko, and Zapechelnyuk (2006), Schipper (2003), Cornes and Hartley (2005), Jensen (2005), and Acemoglu and Jensen (2013).

Definition 4 (Aggregate Taking Strategy) The action profile $\mathbf{a}^{\circ}=\left(a_{1}^{\circ}, \ldots, a_{n}^{\circ}\right)$ with $a_{i}^{\circ} \in$ $A_{i}$ is an optimal aggregate taking outcome of an aggregate strategic game $G=\left\langle N,\left(A_{i}\right),\left(\pi_{i}\right)\right\rangle$ if for all $i \in N$,

$$
\pi_{i}\left(a_{i}^{\circ}, \aleph^{n}\left(\mathbf{a}^{\circ}\right)\right) \geq \pi_{i}\left(a_{i}, \aleph^{n}\left(\mathbf{a}^{\circ}\right)\right) \text {, for all } a_{i} \in A_{i}
$$

It is strict aggregate taking outcome if the inequality holds strictly. We call $a_{i}^{\circ}$ player $i$ 's aggregate taking strategy.

This notion is due to Possanjenikov (2002). Note that an ATS generalizes naturally the competitive outcome or the Walrasian outcome in oligopoly games (see also Vega-Redondo, 1997, Schipper, 2003, Alós-Ferrer and Ania, 2005). Alós-Ferrer and Ania (2005) provide an existence result for games satisfying the single-crossing property based on Topkis (1998), Milgrom and Shannon (1994), and Tarski's fixed-point theorem.

We say that an aggregative game $G$ is (strictly) supermodular if it is supermodular and for each player $i \in N$ the payoff function $\pi_{i}$ has (strictly) increasing differences in $\left(a_{i}, \aleph^{n}(\mathbf{a})\right)$ on $A_{i} \times X$. An aggregative game $G$ is (strictly) submodular if it is (strictly) submodular and for each player $i \in N$ the payoff function $\pi_{i}$ has (strictly) decreasing differences in $\left(a_{i}, \aleph^{n}(\mathbf{a})\right)$ on $A_{i} \times X$. 
We say that an aggregative game $G$ has positive (resp. negative) aggregate externalities if it has positive (resp. negative) externalities and for each player $i \in N$ the payoff $\pi_{i}\left(a_{i}, \aleph^{n}(\mathbf{a})\right.$ ) is increasing (resp. decreasing) in $\aleph^{n}(\mathbf{a})$ for each $a_{i} \in A_{i}$. An aggregative game G has strict positive (resp. negative) aggregate externalities if the strict versions hold.

Lemma 2 Let $G=\left\langle N,\left(A_{i}\right),\left(\pi_{i}\right)\right\rangle$ be an aggregative strategic game with $A_{i}$ being a chain for each $i \in N$, and let $\mathbf{a}^{\circ}$ be a symmetric ATS profile and $\mathbf{a}^{*}$ be a symmetric Nash equilibrium of $G$. If the aggregative game $G$ is such that $\pi_{i}$ satisfies

(i) the decreasing difference in $\left(a_{i}, \aleph^{n}(\mathbf{a})\right)$ on $A_{i} \times X$ and $G$ has strict negative (resp. positive) aggregate externalities, or

(ii) the strict decreasing differences in $\left(a_{i}, \aleph^{n}(\mathbf{a})\right)$ on $A_{i} \times X$ and $G$ has negative (resp. positive) aggregate externalities,

then $\mathbf{a}^{\circ} \unrhd \mathbf{a}^{*}\left(\right.$ resp. $\left.\mathbf{a}^{\circ} \unlhd \mathbf{a}^{*}\right)$.

In the appendix we prove this result more generally for games satisfying the single crossing property (Milgrom and Shannon, 1994).

Example 1 (Continued) Consider again the Cournot duopoly of Example 1. This is an aggregative game with the sum of quantities, $\aleph^{1}\left(a_{1}\right)=a_{1}$ and $\aleph^{2}\left(a_{1}, a_{2}\right)=a_{1}+a_{2}$, as the natural aggregator. We can write the payoff function as $\pi_{i}\left(a_{i}, \aleph^{2}\left(a_{1}, a_{2}\right)\right)=\left(100-\aleph^{2}\left(a_{1}+\right.\right.$ $\left.a_{2}\right) a_{i}-\frac{1}{2} a_{i}^{2}$. This aggregative game is submodular. It has negative aggregate externalities (since the demand function is downward sloping in aggregate quantity). The unique symmetric ATS is the competitive outcome where price equals marginal cost, $a_{i}^{\circ}=\frac{100}{3}$ for $i=1,2$. The unique symmetric Nash equilibrium is $a_{i}^{*}=25$ and thus smaller than the unique symmetric ATS, thus illustrating Lemma 2.

\subsection{Optimism, Pessimism, and Complete Ignorance}

In this section, we model Knightian uncertainty by Choquet expected utility theory with neoadditive capacities, which will be applied to games in the next section. Let $\Omega$ be a space of mutually exclusive states and $\Sigma$ be a corresponding sigma-algebra of events.

Definition 5 (Neo-additive capacity) The ambiguous belief of decision maker $i$ is represented by a neo-additive capacity $\nu: \Sigma \longrightarrow \mathbb{R}_{+}$is defined by $\nu_{i}(E)=\alpha_{i} \delta_{i}+\left(1-\delta_{i}\right) \mu_{i}(E)$, for

all $E \in \Sigma$ s.t. $\emptyset \varsubsetneqq E \varsubsetneqq \Omega, \nu_{i}(\emptyset)=0, \nu_{i}(\Omega)=1, \mu_{i}$ a probability measure on $(\Omega, \Sigma)$, and $\alpha_{i}, \delta_{i} \in[0,1]$.

Chatenauneuf, Eichberger, and Grant (2007) axiomatize Choquet expected utility with neo-additive capacities. When a player's belief is represented by a neo-additive capacity, her Choquet expected utility from an action $a_{i} \in A_{i}$ takes the following form.

Definition 6 (Choquet expected utility with neo-additive capacity) Let $\pi_{i}: A_{i} \times \Omega \longrightarrow$ $\mathbb{R}$ be player $i$ 's payoff function with $\bigcirc_{i}\left(a_{i}\right):=\max _{\omega \in \Omega} \pi_{i}\left(a_{i}, \omega\right)$ and $\boldsymbol{W}_{i}\left(a_{i}\right):=\min _{\omega \in \Omega} \pi_{i}\left(a_{i}, \omega\right)$. 
Player i's Choquet expected utility from an action $a_{i}$ with respect to her neo-additive capacity $\nu_{i}$ is given by

$$
u_{i}\left(a_{i}, \nu_{i}\right)=\delta_{i}\left[\alpha_{i} \oslash_{i}\left(a_{i}\right)+\left(1-\alpha_{i}\right) \mathbb{W}_{i}\left(a_{i}\right)\right]+\left(1-\delta_{i}\right) \mathbb{E}_{\mu_{i}}\left[\pi_{i}\left(a_{i}, \omega\right)\right],
$$

with $\mathbb{E}_{\mu_{i}}\left[\pi_{i}\left(a_{i}, \omega\right)\right]$ being the expected payoff with respect to the probability measure $\mu_{i}$ on $(\Omega, \Sigma)$.

Choquet expected utility with respect to neo-additive capacities is just a special case of Choquet expected utility (Schmeidler, 1989). What makes it useful for our purpose is that the parametrization of neo-additive capacities suggests a differentiation between the amount of ambiguity $\delta_{i}$ faced by decision maker $i$ and her attitude towards this ambiguity $\alpha_{i}$. We call the parameter $\delta_{i}$ the degree of ignorance whereas $\alpha_{i}$ is the degree of optimism.

Definition 7 (Optimism, Pessimism and (Complete) Ignorance) Assume that player $i$ is a Choquet expected utility maximizer with a neo-additive capacity. We interpret $\alpha_{i}^{\prime} \geq \alpha_{i}$ as $\alpha_{i}^{\prime}$ being more optimistic than $\alpha_{i}$ (or $\alpha_{i}$ being more pessimistic than $\alpha_{i}^{\prime}$ ) for a given $\delta_{i}$. We interpret $\delta_{i}^{\prime} \geq \delta_{i}$ as $\delta_{i}^{\prime}$ being more ignorant than $\delta_{i}$ for a given $\alpha_{i}$. We say that $i$ is completely ignorant if $\delta_{i}=1$. We say that $i$ is a realist if $\delta_{i}=0 .{ }^{5}$

Intuitively, a neo-additive capacity describes a situation in which the decision maker $i$ has an additive probability measure $\mu_{i}$ over outcomes but also lacks confidence in this belief. She reacts to this ambiguity with overweighing good or bad outcomes. A decision maker is optimistic (resp. pessimistic) if she overweighs good (resp. bad) outcomes. The latter interpretation is based on Wakker (2001) and justified in our context by Chatenauneuf, Eichberger, and Grant (2007).

CEU with neo-additive capacities entails several familiar decision-theoretic approaches as special cases. Cases 1 to 3 concern decision making under complete ignorance.

1. If $\delta_{i}=1$ and $\alpha_{i}=0$, then the preference has the Minimax form and is extremely pessimistic (Wald, 1951);

2. if $\delta_{i}=1$ and $\alpha_{i}=1$, then the preference has the Maximax form and exhibits a maximal degree of optimism;

3. if $\delta_{i}=1$ and $\alpha_{i} \in[0,1]$, then these preferences coincide with the Hurwicz criterion, (see Hurwicz, 1951, and Arrow and Hurwicz, 1972);

4. if $\delta_{i}=0$ and $\alpha_{i} \in[0,1]$, the belief coincides with a conventional probability measure. In particular, the capacity is additive, i.e., $A, B \subseteq \Omega, A \cap B=\emptyset, \nu_{i}(A \cup B)=\nu_{i}(A)+\nu_{i}(B)$. This is the case of Subjective expected utility (SEU).

\subsection{Strategic Games with Optimism and Pessimism}

A neo-additive capacity $\nu_{i}$ is defined by $\alpha_{i}, \delta_{i}$, and the additive probability measure $\mu_{i}$. Since players in games face strategic uncertainty about opponents' actions, $\mu_{i}$ represents a probabilistic conjecture over opponents' actions. This probability measure is to be determined

\footnotetext{
${ }^{5}$ Note that if $\delta_{i}=0$, then the parameter $\alpha_{i} \in[0,1]$ can be arbitrary.
} 
endogenously in equilibrium. In contrast, $\alpha_{i}$ and $\delta_{i}$ will be treated exogenously for a game. Since in games the payoff function $\pi_{i}$ is fixed, we simply refer to $\left(\alpha_{i}, \delta_{i}\right)$ as the preference of player $i$.

To connect Choquet expected utility to games, we let each player's state-space be the opponents' set of action profiles. I.e., player $i$ 's state-space is $\Omega_{i}=\times_{j \neq i} A_{j}$. We focus here on equilibria in pure strategies only. Hence a player's probability measure over opponents' actions is degenerate as it assigns unit probability to exactly one profile of opponents' actions. Therefore we write for player $i$ 's Choquet expected payoff from an action $a_{i}$ given $i$ 's belief

$$
u_{i}\left(a_{i}, a_{-i}, \alpha_{i}, \delta_{i}\right)=\delta_{i}\left[\alpha_{i} \oslash_{i}\left(a_{i}\right)+\left(1-\alpha_{i}\right) \boldsymbol{\Psi}_{i}\left(a_{i}\right)\right]+\left(1-\delta_{i}\right) \pi_{i}\left(a_{i}, a_{-i}\right) .
$$

For this Choquet expected payoff to be well-defined, we need to require that both $\nabla\left(a_{i}\right)$ and $\mathbf{H}\left(a_{i}\right)$ exist for every $a_{i} \in A_{i}$.

Assumption 1 For each player $i \in N, A_{i}$ is a subcomplete sublattice of a complete lattice $X$ and $\pi_{i}$ is continuous in $a_{-i}$ on $A_{-i}$ for every $a_{i} \in A_{i}$.

Remark 1 Assumption 1 implies that $u_{i}\left(a_{i}, a_{-i}, \alpha_{i}, \delta_{i}\right)$ is well-defined for every $a_{i} \in A_{i}, a_{-i} \in$ $A_{-i}, \alpha_{i}, \delta_{i} \in[0,1]^{2}$.

Assumption 1 implies that the best and worst payoffs exist, and thus function $u_{i}$ is well defined. The proof is identical to the first part of the proof of Lemma 1 in the appendix. Since this is assumption is very basic, we impose this assumption from now on without explicitly mentioning it any further.

Denote by $\boldsymbol{\alpha}=\left(\alpha_{1}, \ldots, \alpha_{n}\right)$ the profile of degrees of optimism and by $\boldsymbol{\delta}=\left(\delta_{1}, \ldots, \delta_{n}\right)$ the profile of degrees of ignorance. Let $G(\boldsymbol{\alpha}, \boldsymbol{\delta})=\left\langle N,\left(A_{i}\right),\left(u_{i}\left(\alpha_{i}, \delta_{i}\right)\right)\right\rangle$ be a "perturbed" strategic game derived from $G$ by replacing $\pi_{i}$ with $u_{i}\left(\alpha_{i}, \delta_{i}\right)$ for all $i \in N$. Note that $G(\boldsymbol{\alpha}, \mathbf{0})=G$ for all $\boldsymbol{\alpha} \in[0,1]^{n}$.

Definition 8 (Equilibrium under Knightian Uncertainty) For a strategic game G, an Equilibrium under Knightian uncertainty $(E K U) \mathbf{a}^{*}(\boldsymbol{\alpha}, \boldsymbol{\delta}) \in \mathbf{A}$ with degrees of ignorance $\boldsymbol{\delta}=$ $\left(\delta_{1}, \ldots, \delta_{n}\right)$ and degrees of optimism $\boldsymbol{\alpha}=\left(\alpha_{1}, \ldots, \alpha_{n}\right)$ is a pure Nash equilibrium of the game $G(\boldsymbol{\alpha}, \boldsymbol{\delta})$. I.e., for all $i \in N$,

$$
u_{i}\left(a_{i}^{*}(\boldsymbol{\alpha}, \boldsymbol{\delta}), a_{-i}^{*}(\boldsymbol{\alpha}, \boldsymbol{\delta}), \alpha_{i}, \delta_{i}\right) \geq u_{i}\left(a_{i}(\boldsymbol{\alpha}, \boldsymbol{\delta}), a_{-i}^{*}(\boldsymbol{\alpha}, \boldsymbol{\delta}), \alpha_{i}, \delta_{i}\right), \text { for all } a_{i} \in A_{i} .
$$

Equilibrium under Knigthian uncertainty, as here defined, is just pure Nash equilibrium of the strategic game defined with Choquet expected utility function featuring neo-additive capacities. It implies equilibrium under ambiguity of Eichberger, Kelsey, and Schipper (2009); see their Proposition 1. It also implies equilibrium in beliefs under ambiguity of Eichberger and Kelsey (2014), who allow for optimism and pessimism as well by using Choquet expected utility with more general JP-capacities (Jaffray and Philippe, 1997). When restricted to neo-additive capacities, their support notion of capacities coincides with the support of the probability measure of neo-additive capacities (Eichberger and Kelsey, 2014, Proposition 3).

(Strict) Supermodularity or submodularity of $G$ is preserved under "perturbations" with Knightian uncertainty as modelled by Choquet expected utility with neo-additive capacities. 
Lemma 3 If $G$ is (strictly, resp.) supermodular, then $G(\boldsymbol{\alpha}, \boldsymbol{\delta})$ is (strictly, resp.) supermodular for any $(\boldsymbol{\alpha}, \boldsymbol{\delta}) \in[0,1]^{n} \times[0,1]^{n}$. The analogous result holds for $G$ being (strictly, resp.) submodular.

The proof is contained in the appendix.

Often the literature makes use of a weaker ordinal notion of strategic complementarity. The ordinal version of supermodularity, quasisupermodularity (see Milgrom and Shannon, 1994), may not need to be preserved under Knightian uncertainty since the sum of two quasisupermodular functions does not need to be quasisupermodular unless either is supermodular (Topkis, 1998, pp. 62). An analogous conclusion holds for functions satisfying the ordinal version of increasing (resp. decreasing) differences, the (resp. dual) single crossing property (see Milgrom and Shannon, 1994).

Given the game $G(\boldsymbol{\alpha}, \boldsymbol{\delta})$, let player $i$ 's best response correspondence be defined by

$$
b_{i}\left(a_{-i}, \alpha_{i}, \delta_{i}\right):=\left\{a_{i} \in A_{i}: u_{i}\left(a_{i}, a_{-i}, \alpha_{i}, \delta_{i}\right) \geq u_{i}\left(a_{i}^{\prime}, a_{-i}, \alpha_{i}, \delta_{i}\right) \text {, for all } a_{i}^{\prime} \in A_{i}\right\} .
$$

Lemma 4 If $G$ is supermodular (resp. submodular), then for any $i \in N$ and any $\left(\alpha_{i}, \delta_{i}\right) \in$ $[0,1]^{2}$, the best response $b_{i}\left(a_{-i}, \alpha_{i}, \delta_{i}\right)$ is a sublattice of $A_{i}$ and increasing (resp. decreasing) in $a_{-i}$ on $\left\{a_{-i} \in A_{-i}: b_{i}\left(a_{-i}, \alpha_{i}, \delta_{i}\right) \neq \emptyset\right\}$.

The proof is contained in the appendix.

If the Hurwicz criterion is satisfied, i.e., the player is completely ignorant, then her objective function does not depend on the opponents' actions. This leads to the following observation:

Remark 2 If $\delta_{i}=1$, then $b_{i}\left(a_{-i}, \alpha_{i}, 1\right)$ is trivially constant in $a_{-i}$ on $A_{-i}$ for any $\alpha_{i} \in[0,1]$.

Example 1 (Continued) Consider again Example 1. Let $A=[0,50]$. We have $\varnothing\left(a_{i}\right)=$ $\pi\left(a_{i}, 0\right)$ and $\left(a_{i}\right)=\pi\left(a_{i}, 50\right)$. Thus, assuming CEU given player $i$ 's degree of optimism $\alpha_{i}$ and degree of ignorance $\delta_{i}$, player $i$ 's Choquet expected utility from quantity $a_{i}$ when opponent $3-i$ selects quantity $a_{3-i}$ is $u\left(a_{i}, a_{3-i}, \alpha_{i}, \delta_{i}\right)=\delta_{i}\left[\alpha_{i} \pi\left(a_{i}, 0\right)+\left(1-\alpha_{i}\right) \pi\left(a_{i}, 50\right)\right]+\left(1-\delta_{i}\right) \pi\left(a_{i}, a_{3-i}\right)$. Player $i$ 's best response to $a_{3-i}$ is given by $b\left(a_{3-i}, \alpha_{i}, \delta_{i}\right)=\frac{100+50 \alpha_{i} \delta_{i}-\delta_{i}\left(50-a_{3-i}\right)-a_{3-i}}{3}$. If $\delta_{i}=1$, then player $i$ 's best response is constant in $a_{3-i}$. Given $\left(\alpha_{1}, \alpha_{2}, \delta_{1}, \delta_{2}\right)$, the EKU is given by $a_{i}^{*}\left(\alpha_{1}, \alpha_{2}, \delta_{1}, \delta_{2}\right)=\frac{200+50 \alpha_{3-i} \delta_{3-i} \delta_{i}-50 \alpha_{3-i} \delta_{3-i}-50 \delta_{3-i} \delta_{i}+150 \alpha_{i} \delta_{i}+50 \delta_{3-i}-50 \delta_{i}}{8-\delta_{3-i} \delta_{i}+\delta_{3-i}+\delta_{i}}$. Clearly, EKU coincides with Nash equilibrium if there is no ignorance, i.e., $\delta_{1}=\delta_{2}=0$.

We are able to state general results on the existence of equilibrium under Knightian uncertainty. The proof (in the appendix) of the following proposition relies on a generalization of Tarski's fixed-point theorem by Zhou (1994).

Proposition 1 (Existence in Supermodular Games) If $G$ is supermodular and for all $i \in$ $N, A_{i}$ is a non-empty complete lattice, and $\pi_{i}$ is upper semicontinuous on $A_{i}$ for every $a_{-i} \in A_{-i}$ and continuous on $A_{-i}$ for every $a_{i} \in A_{i}$, then for any $(\boldsymbol{\alpha}, \boldsymbol{\delta}) \in[0,1]^{n} \times[0,1]^{n}$ the set of equilibria under Knightian uncertainty is a complete lattice and a greatest and least equilibrium exist. 
Since there is no general fixed-point theorem applicable to decreasing best responses, we use for the next result a different approach to existence for submodular games based on Kukushkin (1994) and Novshek (1985).

Proposition 2 (Existence in Submodular Games) If $G$ is submodular and for all $i \in N$, $A_{i}$ is a non-empty compact subset of $\mathbb{R}_{+}, \pi_{i}$ is defined on $A_{i}$ and the range of sums of opponents actions, and is continuous in both variables, then for any $(\boldsymbol{\alpha}, \boldsymbol{\delta}) \in[0,1]^{n} \times[0,1]^{n}$ the set of equilibria under Knightian uncertainty is non-empty.

The proof is contained in the appendix. Kukushkin (1994) requires for each player a realvalued compact action set, an upper hemi-continuous best response correspondence with a single-valued selection that is decreasing in the additive aggregate of opponents' actions. The last assumption is slightly weaker than decreasing best responses in the additive aggregate of opponents' actions that result from decreasing differences of the Choquet expected payoffs in $\left(a_{i}, a_{-i}\right)$ on $\mathbf{A}$ in our case. The pseudo-potential approach to existence by Dubey, Haimanko and Zapechelnyuk (2006) would be an alternative to Kukushkin (1994). This approach could be used for games both with increasing and decreasing best responses. Our existence theorem may be generalized to action sets that are compact subsets of a partially ordered topological vector-space and more general continuous aggregators of opponents' actions using results by Jensen (2005).

In the later analysis, complete ignorance plays a prominent role. For this special case, no matter whether the game is supermodular or submodular, existence and uniqueness is rather straight-forward (see the appendix for brief proofs).

Proposition 3 (Existence under Complete Ignorance) Let $G=\left\langle N,\left(A_{i}\right),\left(\pi_{i}\right)\right\rangle$ be a strategic game with for all $i \in N, A_{i}$ being a non-empty complete lattice, $\pi_{i}$ being upper semincontinuous and supermodular on $A_{i}$ for every $a_{-i} \in A_{-i}$ and continuous on $A_{-i}$ for every $a_{i} \in A_{i}$, and the Hurwicz criterion satisfied (i.e., $\delta_{i}=1$ for all $i \in N$ ). Then for all $\boldsymbol{\alpha} \in[0,1]^{n}$, the set of equilibria under Knightian uncertainty is a complete lattice and a greatest and least equilibrium exist.

Proposition 4 (Uniqueness under Complete Ignorance) Let $G=\left\langle N,\left(A_{i}\right),\left(\pi_{i}\right)\right\rangle$ be a strategic game with for all $i \in N, A_{i} \subseteq \mathbb{R}^{m_{i}}$ for some positive natural number $m_{i}$, $\pi_{i}$ being strictly concave on $A_{i}$, and the Hurwicz criterion being satisfied (i.e., $\delta_{i}=1$ ). If there exists an equilibrium under Knightian uncertainty for $\boldsymbol{\alpha} \in[0,1]^{n}$, then it is unique and each player's equilibrium action is her strictly dominant action.

\subsection{Monotone Comparative Statics}

To analyze the effect of mutants with respect to the degrees of optimism and ignorance, $\alpha_{i}$ and $\delta_{i}$, respectively, in equilibrium under Knightian uncertainty, it will be helpful to study first the monotone comparative statics of equilibrium under Knightian uncertainty with respect to these parameters. Given the level of generality, these results may be of interest in their own right. 
Proposition 5 If $\pi_{i}$ is supermodular in $a_{i}$ on $A_{i}$, has increasing differences in $\left(a_{i}, a_{-i}\right)$ on $\mathbf{A}$ and has positive (resp. negative) externalities, then $b_{i}\left(a_{-i}, \alpha_{i}, \delta_{i}\right)$ is increasing (resp. decreasing) in $\alpha_{i}$ on $\left\{\alpha_{i} \in[0,1]: b_{i}\left(a_{-i}, \alpha_{i}, \delta_{i}\right) \neq \emptyset\right\}$ for each $\delta_{i} \in[0,1]$. The same comparative statics obtains if $\pi_{i}$ has decreasing differences in $\left(a_{i}, a_{-i}\right)$ on $\mathbf{A}$ and negative (resp. positive) externalities. If $\pi_{i}$ has strictly increasing differences in $\left(a_{i}, a_{-i}\right)$ on $\mathbf{A}$ and positive (resp. negative) externalities, $\alpha_{i}^{\prime \prime}>\alpha_{i}^{\prime}$ in $[0,1]$, given $\delta_{i} \in[0,1]$, and for any $a_{-i} \in A_{-i}, a_{i}^{\prime} \in b_{i}\left(a_{-i}, \alpha_{i}^{\prime}, \delta_{i}\right)$ and $a_{i}^{\prime \prime} \in b_{i}\left(a_{-i}, \alpha_{i}^{\prime \prime}, \delta_{i}\right)$, then $a_{i}^{\prime} \unlhd(\unrhd) a_{i}^{\prime \prime}$. In this case, if one picks any $a_{i}\left(\alpha_{i}\right)$ in $b_{i}\left(a_{-i}, \alpha_{i}, \delta_{i}\right)$ for each $\alpha_{i}$ with $b_{i}\left(a_{-i}, \alpha_{i}, \delta_{i}\right)$ nonempty, then $a_{i}\left(\alpha_{i}\right)$ is increasing (resp. decreasing) in $\alpha_{i}$ on $\left\{\alpha_{i} \in[0,1]: b_{i}\left(a_{-i}, \alpha_{i}\right) \neq \emptyset\right\}$. The same comparative statics obtains if $\pi_{i}$ has strictly decreasing differences in $\left(a_{i}, a_{-i}\right)$ on $\mathbf{A}$ and negative (resp. positive) externalities.

The proof in the appendix proceeds in two steps. First, we show that increasing differences in own and opponents' actions and negative externalities are sufficient for increasing differences of Choquet expected utility with respect to own actions and the degree of optimism (and analogous for the dual notions). The intuition is as follows: Increasing differences in own and opponents' actions means that the marginal benefit from an increase of my action is increasing in opponents' actions. With positive externalities, the best outcome of player $i$ is reached with the largest actions of opponents. Thus, increasing optimism is like putting more weight on the largest actions of opponents, which increases the marginal benefit of the player's own action. In a second step we observe the usual monotone comparative statics (Topkis, 1998), namely that when a supermodular function has increasing differences in an action and a parameter, then the argmax correspondence (of the perturbed game) is increasing in the parameter (for where it is nonempty). Proposition 5 follows now as a corollary.

Together with general results of Topkis (1998, Theorem 4.2.2) on supermodular games, the previous observations on the monotone comparative statics of best responses in the perturbed game with respect to the degree optimism imply immediately monotone comparative statics of equilibrium under Knigthian uncertainty with respect to the degree of optimism in supermodular games.

Proposition 6 (Mon. Comp. Statics - Supermodularity) If $G$ is a supermodular game with positive (resp. negative) externalities, then the greatest and the least equilibrium under Knightian uncertainty are increasing (resp. decreasing) in optimism and decreasing (resp. increasing) in pessimism.

As usual in the literature, there is no dual result on the monotone comparative statics for submodular games. However, if the Hurwicz criterion is satisfied (i.e., under complete ignorance), then we can derive a dual but more special result for submodular games with externalities. It makes use of the fact that under complete ignorance the best response correspondence is constant in opponents' actions.

Proposition 7 (Mon. Comp. Statics - Submodularity and Complete Ignorance) If $G$ is a submodular game with negative (resp. positive) externalities and the Hurwicz criterion $\left(\delta_{i}=1\right)$ is satisfied for all players $i \in N$, then the greatest and the least equilibrium under Knightian uncertainty are increasing (resp. decreasing) in optimism and decreasing (resp. increasing) in pessimism. 
The proof is contained in the appendix.

Example 1 (Continued) The last observation can be illustrated with Example 1. Assume complete ignorance of both players, $\delta_{1}=\delta_{2}=1$. For simplicity, assume further that $\alpha_{1}=\alpha_{2} \equiv \alpha$. The corresponding EKU is given by $a_{i}^{*}(\alpha, 1)=\frac{50(1+\alpha)}{3}$ for $i=1,2$. Clearly, this is increasing in $\alpha \in[0,1]$.

We emphasize that although complete ignorance implies that best responses are constant in opponents' actions (Remark 2), it does not mute the monotone comparative statics w.r.t. the degree of optimism. The degree of optimism affects which constant action is a best response no matter whether we focus on sub- or supermodular aggregative games. In the former class, it even facilitates the monotone comparative statics w.r.t. the degree of optimism because for submodular games such results would be elusive without complete ignorance.

\section{Evolutionary Stable Neo-additive CEU-Preferences}

In this section, we restrict the analysis to symmetric games. This is natural in an evolutionary context where relative payoffs are relevant. Because of symmetry, we write for all $i \in N$, $A_{i}=A$ and $\pi_{i}(\mathbf{a})=\pi\left(a_{i} \mid a_{-i}\right)$. Note that symmetry implies $\varnothing_{i}=\varnothing$ and $\boldsymbol{\Psi}_{i}=$ Furthermore, we assume that each player's set of actions $A$ is a chain. This is because we do not know how to assess the equilibrium payoff of a mutant playing an action that is incomparable to a non-mutant's action.

\subsection{Notion of Evolutionary Stability}

Denote by $T_{G}$ an arbitrary collection of any player's preferences that can possibly be defined in a game form $\langle N, A\rangle$ of the strategic game $G=\langle N, A, \pi\rangle$. For instance, we could parameterize player $i$ 's Choquet expected utility functions over outcomes in the game $G$ with respect to neo-additive capacities by $t_{i}=\left(\alpha_{i}, \delta_{i}\right) \in T_{G}=[0,1]^{2}$ (for a fixed payoff function $\pi$ ). Yet we can allow here for an even more general set of preferences like preferences over other players' payoffs, beliefs etc. The only requirement we impose is that $T_{G}$ includes Choquet expected utility functions over outcomes of the strategic game $G$ with respect to neo-additive capacities and the fixed payoff function $\pi$ as discussed in Section 2.4.

Let us denote a profile of all players' preferences by $\mathbf{t} \in \mathbf{T}_{G}$. Let $G(\mathbf{t})$ be the strategic game played when $\mathbf{t}$ is the profile of players' preferences in the game form $\langle N, A\rangle$. Further, let $\mathcal{E}(G(\mathbf{t}))$ be the set of pure strategy equilibria given the game $G(\mathbf{t})$. Since we allow the collection of preferences to be arbitrary, equilibrium is not well-defined. The only requirement we impose is that all Choquet expected utility players with neo-additive capacities play their best response to opponents' equilibrium actions. ${ }^{6}$ This implies that if all players are Choquet expected utility maximizers with neo-additive capacities, then an equilibrium is defined by

\footnotetext{
${ }^{6}$ There is no reason to assume that with an arbitrary collection of preferences there is a meaningful notion of equilibrium. However, we show below, that complete ignorance is evolutionary stable. For such players the equilibrium notion considered here makes sense. Such players ignore other players and just play their individually preferred action no matter what others do.
} 
equilibrium under Knightian uncertainty (Definition 8). It also implies that if all players are expected utility maximizers, then an equilibrium is defined by Nash equilibrium of the game with material payoffs.

We restrict the set of abstract equilibria further by focusing on intra-group symmetric equilibria defined by

$$
\mathcal{E}^{\text {sym }}(G(\mathbf{t})):=\left\{\mathbf{a}(\mathbf{t}) \in \mathcal{E}(G(\mathbf{t})): t_{i}=t_{j} \text { implies } a_{i}(\mathbf{t})=a_{j}(\mathbf{t})\right\} .
$$

This is the set of equilibria in which players with the same preference play also the same action. Since for an abstract set of preferences, existence of such equilibria can not be guarantied, we assume $\mathcal{E}^{\text {sym }}(G(\mathbf{t})) \neq \emptyset$ for all $\mathbf{t} \in \mathbf{T}_{G}$. Since we did not specify what players would play in equilibrium except for players with Choquet expected utility preferences with respect to neoadditive capacities, nonemptyness is not a strong assumption. When all players are Choquet expected utility players with respect to neo-additive capacities, our existence results apply (for the class of games that we consider here). An alternative interpretation of our setting is that we restrict $\mathbf{T}_{G}$ to Choquet expected utility with respect to neo-additive capacities only for which our existence results guarantee nonemptyness of $\mathcal{E}^{\text {sym }}(G(\mathbf{t}))$.

As standard in the literature on the evolution of preferences, each player $i$ chooses according to her ex-ante objective function indexed by $t_{i}$. For instance, if $i$ is a Choquet expected utility maximizer, then she chooses according to her Choquet expected utility function. However, the player's fitness is evaluated by her material payoff $\pi$. This conforms for instance to fitness considerations in business or academia. Ultimately the success of a manager (resp. assistant professor) is assessed by her realized profit (resp. publications) and not by what the manager (resp. assistant professor) originally expected.

In many economic situations such as in markets with imperfect competition, the provision of public goods etc., a finite number of players interact repeatedly in some strategic context. For such environments, Schaffer $(1988,1989)$ introduced a notion of evolutionary stable strategy. It is an extension of the standard evolutionary stable strategy for large populations to finite populations, in which each player plays against all other players ("playing-the-field"). An action $a \in A$ is a finite population evolutionary stable strategy (fESS) of a symmetric strategic game $G=\langle N, A, \pi\rangle$ if

$$
\pi\left(a \mid a^{\prime}, a, \ldots, a\right) \geq \pi\left(a^{\prime} \mid a, \ldots, a\right) \text { for all } a^{\prime} \in A .
$$

We apply Schaffer's notion of finite population ESS to the evolution of preferences in aggregative games. As convention we denote by $j$ the mutant and by $i \neq j$ a non-mutant. For any preference $t \in T_{G}$ and mutant $j$, we denote $t_{-j}=(\overbrace{t, \ldots, t}^{n-1})$.

Definition 9 (fESP) $t \in T_{G}$ is a Finite Population Evolutionary Stable Preference (fESP) in a symmetric aggregative game $G=\langle N, A, \pi\rangle$ if for all mutant prefences $t^{\prime} \in T_{G}$,

$$
\pi\left(a_{i}^{*}\left(t_{j}^{\prime}, t_{-j}\right), \aleph^{n}\left(\mathbf{a}^{*}\left(t_{j}^{\prime}, t_{-j}\right)\right)\right) \geq \pi\left(a_{j}^{*}\left(t_{j}^{\prime}, t_{-j}\right), \aleph^{n}\left(\mathbf{a}^{*}\left(t_{j}^{\prime}, t_{-j}\right)\right)\right)
$$

for some $\mathbf{a}^{*}\left(t_{j}^{\prime}, t_{-j}\right) \in \mathcal{E}^{s y m}\left(G\left(t_{j}^{\prime}, t_{-j}\right)\right)$.

A finite population evolutionary stable preference $t \in T_{G}$ is robust (RfESP) in a symmetric aggregative game $G$ if for all mutant preferences $t^{\prime} \in T_{G}$ inequality (8) holds for all $\mathbf{a}^{*}\left(t_{j}^{\prime}, t_{-j}\right) \in$ $\mathcal{E}^{s y m}\left(G\left(t_{j}^{\prime}, t_{-j}\right)\right)$. 
A finite population evolutionary stable preference $t \in T_{G}$ is globally stable (GfESP) in a symmetric aggregative game $G$ if for all mutant preferences $t^{\prime} \in T_{G}$,

$\pi\left(a_{i}^{*}(\overbrace{t^{\prime}, \ldots, t^{\prime}}^{m}, t, \ldots, t), \aleph^{n}\left(\mathbf{a}^{*}(\overbrace{t^{\prime}, \ldots, t^{\prime}}^{m}, t, \ldots, t)\right)\right) \geq \pi\left(a_{j}^{*}(\overbrace{t^{\prime}, \ldots, t^{\prime}}^{m}, t, \ldots, t), \aleph^{n}\left(\mathbf{a}^{*}(\overbrace{t^{\prime}, \ldots, t^{\prime}}^{m}, t, \ldots, t)\right)\right)$,

for some $\mathbf{a}^{*}(\overbrace{t^{\prime}, \ldots, t^{\prime}}^{m}, t, \ldots, t) \in \mathcal{E}^{s y m}\left(G(\overbrace{t^{\prime}, \ldots, t^{\prime}}^{m}, t, \ldots, t)\right)$ for all $m \in\{1, \ldots, n-1\}$.

A finite population evolutionary stable preference $t \in T_{G}$ is robust globally stable in a symmetric aggregative game $G$ if for all mutant preference $t^{\prime} \in T_{G}$ inequality (9) holds for all $\mathbf{a}^{*}(\overbrace{t^{\prime}, \ldots, t^{\prime}}^{m}, t, \ldots, t) \in \mathcal{E}^{s y m}\left(G(\overbrace{t^{\prime}, \ldots, t^{\prime}}^{m}, t, \ldots, t)\right)$ for all $m \in\{1, \ldots, n-1\}$.

In words, a preference is evolutionary stable if there is an equilibrium in which the player with such a preference is more successful than any mutant. A preference is robust evolutionary stable if the player with such a preference is more successful than any mutant in any equilibrium. Finally, a preference is globally evolutionary stable if for any number of mutants there is an equilibrium in which a player with such a preference is more successful than any mutant.

We say that complete ignorance is a finite population evolutionary stable preference in $G$ if the Choquet expected utility function $t=u(\alpha, \delta)$ with $\delta=1$ is a finite population evolutionary stable preference in $G$. That is, the finite population evolutionary stable preference satisfies the Hurwicz criterion and hence involves complete ignorance. Analogous definitions apply to complete ignorance being a (robust) globally stable preference.

We say that a preference with pessimism and complete ignorance is finite population evolutionary stable in $G$ if the Choquet expected utility function $t=u(\alpha, \delta)$ with $\delta=1$ and $\alpha \leq$ $\min \left\{\alpha^{\prime} \in[0,1]: \mathbf{a}\left(\boldsymbol{\alpha}^{\prime},(1, \ldots, 1)\right) \in \mathcal{E}^{s y m}\left(G\left(\boldsymbol{\alpha}^{\prime},(1, \ldots, 1)\right) \cap \mathcal{E}^{s y m}(G)\right\}\right.$ is a finite population evolutionary stable preference in $G$. We say that a preference with optimism and complete ignorance is finite population evolutionary stable in $G$ if the Choquet expected utility function $t=u(\alpha, \delta)$ with $\delta=1$ and $\alpha \geq \max \left\{\alpha^{\prime} \in[0,1]: \mathbf{a}\left(\boldsymbol{\alpha}^{\prime},(1, \ldots, 1)\right) \in \mathcal{E}^{s y m}\left(G\left(\boldsymbol{\alpha}^{\prime},(1, \ldots, 1)\right) \cap \mathcal{E}^{s y m}(G)\right\}\right.$ is a finite population evolutionary stable preference in $G$. (Robust) globally stable preferences with optimism (resp. pessimism) and complete ignorance are defined analogously. These definitions capture the intuition that a preference leading to Nash equilibrium can not be behaviorally distinguished from a realist in equilibrium, i.e., we use symmetric Nash equilibria as the "realism" benchmarks. We call a preference with complete ignorance optimistic if it involves a higher degree of optimism than any symmetric preference profile with complete ignorance that would lead to a symmetric Nash equilibrium. Note that $\mathcal{E}^{\text {sym }}\left(G\left(\boldsymbol{\alpha}^{\prime},(1, \ldots, 1)\right) \cap \mathcal{E}^{s y m}(G)\right.$ is nonempty if there is a symmetric Nash equilibrium $\mathbf{a} \in \mathcal{E}^{\operatorname{sym}}(G)$ for which there exist a symmetric equilibrium under Knightian uncertainty $\mathbf{a}(\boldsymbol{\alpha}, \boldsymbol{\delta})$ of $G$ with $\delta=1$ for all players such that $\mathbf{a}=\mathbf{a}(\boldsymbol{\alpha}, \boldsymbol{\delta})$. Otherwise, if there is no such "realism" benchmark given by plain Nash equilibrium that could be also reached with complete ignorance, then the "optimism" (resp. pessimism) property of complete ignorance preferences might be trivially satisfied. However, Example 1 below shows that this property is meaningfully satisfied in for instance a standard Cournot oligopoly.

We say that the preference with extreme optimism (resp. pessimism) and complete ignorance is finite population evolutionary stable in $G$ if $t=(\alpha, \delta)$ with $\alpha=1$ (resp. $\alpha=0$ ) and $\delta=1$ is an evolutionary stable preference in $G$ (likewise for robust global evolutionary stability). 


\subsection{Submodular Games, Ignorance, and Optimism}

Assumption 2 For any symmetric optimal Aggregative Taking Strategy (ATS) profile $\mathbf{a} \in \mathbf{A}$ of the game $G$ let there exist a symmetric equilibrium under Knightian uncertainty $(E K U) \mathbf{a}(\boldsymbol{\alpha}, \boldsymbol{\delta})$ of $G$ with complete ignorance, $\delta_{i}=1$, for all $i \in N$ such that $\mathbf{a}=\mathbf{a}(\boldsymbol{\alpha}, \boldsymbol{\delta})$.

The assumption says that for any symmetric optimal aggregate taking strategy, there should exist a capacity that satisfies the Hurwicz criterion such that every player maximizing individually her Choquet expected payoffs with respect to this capacity leads to an equilibrium under Knightian uncertainty that equals to the optimal aggregate taking strategy.

The Hurwicz expectation is a weighted average of the best and worst expectations, which when coupled with the assumption of aggregate externalities corresponds to the anticipation of the largest and lowest aggregate of actions. The assumption says that the ATS action can be reached by maximizing a weighted average of the best and worst expectations. That is, to reach an optimal outcome in which the player does not perceive any influence on the aggregate it is sufficient to maximize a suitably weighted average of the best and worst expectations. The assumption is violated if for example there exists a pure action that dominates any other action, and the aggregate taking strategy is different from the Nash equilibrium. Then clearly for any aggregate of opponents' actions, the dominant action would be the best response to any opponents' actions. This is then also the Nash equilibrium action as well as the equilibrium action in any equilibrium under Knightian uncertainty since Knightian uncertainty respects dominance. If the dominant Nash equilibrium action is different from any ATS, then there can not exist a best response equivalent to an ATS. Examples 1 to 3 below satisfy Assumption 2 . Example 4 illustrates what happens if the assumption is violated.

Proposition 8 Let $G=\langle N, A, \pi\rangle$ be a symmetric strict submodular aggregative game with aggregate externalities and $T_{G}$ be a collection of preferences that includes all Hurwicz preferences. Suppose that Assumption 2 holds and that a symmetric optimal aggregate taking strategy (ATS) exists. Then we conclude the following:

(i) There exists a preference with optimism and complete ignorance (a Hurwicz preference) that is globally evolutionary stable in $G$.

(ii) The symmetric equilibrium under Knightian uncertainty resulting from play with this globally evolutionary stable preference profile equals to a symmetric ATS profile.

(iii) If $\pi$ is strictly concave in the player's own action $a_{i} \in A$ for all $a_{-i} \in A_{-i}$, then this preference with optimism and complete ignorance is also a robust globally evolutionary stable preference.

The proof is contained in the appendix.

The intuition of the result is as follows: Under the conditions of Proposition 8, we can find a sufficiently high degree of optimism of such players with complete ignorance and this degree of optimism play exactly the ATS strategy. The ATS strategy is fESS in this class of games. To see this note that in this class of games the ATS strategy is larger than the Nash equilibrium strategy (Lemma 2). If players play Nash equilibrium, then one player playing a somewhat 
larger action decreases her material payoff but decreases the material payoffs of other players even more. This means that playing somewhat larger than Nash equilibrium is relative payoff improving (although not absolute payoff improving). The ATS is exactly the outcome with no relative payoff improving deviations from it signifying the fact that it is fESS. But this implies that the CEU preferences with ignorance that generate this ATS action are fESP.

In Proposition 8, complete ignorance (and thus the Hurwicz criterion) together with a certain degree of optimism is a sufficient condition for finite population evolutionary stability of a preference. This poses the question whether complete ignorance is also necessary. In Example 1 we present a standard Cournot duopoly with linear demand and convex cost where complete ignorance and extreme optimism is the unique preference with an equilibrium under Knightian uncertainty that equals the ATS profile and hence implies GfESS. However, in Example 2 we show in a Cournot duopoly with multiplicative aggregation that complete ignorance and thus the Hurwicz criterion is not necessary for finite population evolutionary stability of a preference. Note that complete ignorance allows us to use arguments of Proposition 7, i.e., a result on the monotone comparative statics of equilibrium under Knightian uncertainty for submodular games with externalities. Otherwise, without complete ignorance, changes in equilibrium under Knightian uncertainty may be ambiguous. This suggests that a full characterization of all finite population evolutionary stable degrees of ignorance and optimism may be elusive within this general approach.

Example 1 (Continued) In this game, the only degree of ignorance and optimism for which the EKU equals the ATS profile is complete ignorance and extreme optimism, i.e., $\delta_{i}=1$ and $\alpha_{i}=1$. Any lower degree of ignorance would require a larger degree of optimism in order to reach ATS, which is impossible. Since for the class of games under consideration, an ATS implies fESS (Schipper, 2003, Alós-Ferrer and Ania, 2005), and an EKU corresponding to an ATS involves complete ignorance, we can conclude that extreme optimism and complete ignorance is a GfESP.

Example 2 (Cournot duopoly with multiplicative aggregate) This example is to show that complete ignorance is not necessary in Proposition 8. Consider two players, $i=1$, 2 , and symmetric payoff functions $\pi\left(a_{i}, a_{3-i}\right)=\left(100-a_{i} a_{3-i}\right) a_{i}-\frac{1}{2} a_{i}^{2}$ with actions $a_{i}$ being in a suitable positive real-valued interval. This is a Cournot duopoly with a multiplicative aggregate of actions. It has decreasing differences and negative aggregate externalities. There exists an unique Nash equilibrium and an ATS. One can compute that $\delta_{i}=1$ and $\alpha_{i}=0.95244$ leads to a unique EKU that equals the ATS. Thus Assumption 1 holds. Since it involves complete ignorance, this is sufficient to conclude that $\left(\delta_{i}, \alpha_{i}\right)$ is an GfESP. Are there other combinations of $\delta_{i}$ and $\alpha_{i}$ that are fESP? It can be verified that indeed there are finite population evolutionary stable preferences without complete ignorance, for instance $\delta_{i}=\frac{1}{2}$ and $\alpha_{i}=1$. Hence complete ignorance is not a necessary condition in Proposition 8.

Although Example 2 shows that complete ignorance is not necessary, Example 1 demonstrates that the class of games where complete ignorance is uniquely evolutionary stable is not negligible. The reason is that Example 1 is not non-generic. The arguments of Example 1 apply even if we slightly perturb the intercept of the inverse demand function. 


\subsection{Supermodular Games, Ignorance, and Pessimism}

Supermodular games have often multiple equilibria. The following assumption guarantees that there are at least two equilibria in pure strategies if $\max A \neq \min A$, i.e., if the set of actions is non-trivial. In particular, the largest and the smallest profile of actions are Nash equilibria.

Assumption 3 Let for all $i \in N, \pi_{i}\left(a_{i}, \inf A_{-i}\right)$ be decreasing in $a_{i} \in A$ and $\pi_{i}\left(a_{i}, \sup A_{-i}\right)$ be increasing in $a_{i} \in A .^{7}$

Note that by Milgrom and Roberts (1990, Theorem 5), for any supermodular game there exist both a largest and smallest profile of actions that survive iterated deletion of strictly dominated strategies, and those combinations of actions are pure Nash equilibria. It is known that only iteratively undominated actions are rationalizable. Assumption 3 can be interpreted as focusing on the subset of actions that can not be eliminated by iterated elimination of strictly dominated actions. It is immediate that if an action is strictly dominated, then it can not be an equilibrium action under Knightian uncertainty for any degree of optimism and ignorance. However, the assumption is not without loss of generality because the set of rationalizable actions with Choquet expected utility preferences is a weak superset of rationalizable actions with expected utility preferences (Dominiak and Schipper, 2019). In Example 4 we illustrate what may happen if Assumption 3 is violated.

Proposition 9 Let $G=\langle N, A, \pi\rangle$ be a symmetric strict supermodular aggregative game with aggregate externalities and $T_{G}$ a collection of preferences that includes the Minimax preference. Suppose that Assumptions 2 and 3 hold. Then we conclude the following:

(i) The preference with extreme pessimism and complete ignorance (the Minimax preference) is finite population evolutionary stable in $G$.

(ii) A symmetric equilibrium under Knightian uncertainty (EKU) resulting from play with this finite population evolutionary stable preference profile equals to a symmetric optimal aggregate taking strategy (ATS).

(iii) If $\pi$ is strictly quasi-concave in the player's own action $a_{i} \in A$ for all $a_{-i} \in A_{-i}$, then this preference with extreme pessimism and complete ignorance (the Minimax preference) is a robust finite population evolutionary stable preference.

The proof is contained in the appendix.

The intuition for the result is as follows: Under the assumptions of Proposition 9, the game has boundary Nash equilibria. Note that these equilibria can be ranked for each player. Depending on the type of externalities, the worse one of those boundary Nash equilibria implies fESS. Moreover, in this class of games, fESS implies ATS. This ATS is also played with Minimax preferences. With Minimax preferences, the player considers the worst action of the opponent.

\footnotetext{
${ }^{7}$ The proof of Proposition 9 uses only the assumption that if $G$ has positive (resp. negative) externalities, then $\pi_{i}\left(a_{i}, \inf A_{-i}\right)$ is decreasing in $a_{i} \in A\left(\operatorname{resp} . \pi_{i}\left(a_{i}, \sup A_{-i}\right)\right.$ is increasing in $\left.a_{i} \in A\right)$. This is slightly weaker than Assumption 3.
} 
Contrary to Proposition 8, we can not show global stability for supermodular games. If such a result were to hold, it would be sufficient to show it in games with positive (resp. negative) externalities for which the smallest (resp. largest) combination of actions is a Nash equilibrium (see Lemma 8 in the appendix). Yet, in such games it is not necessarily the case that the Nash equilibrium is robust against deviations by any proper subcoalitions of players.

In order to show that complete ignorance and extreme pessimism is a robust finite population evolutionary stable preference, we require that $\pi$ is just strictly quasiconcave in the player's own action $a_{i}$ instead of being strictly concave as in Proposition 8. This is due to extreme pessimism and complete ignorance, such that the Choquet expected payoff is equivalent to the worst-case payoff only. Contrary, in Proposition 8 the Choquet expected payoff is a weighted average of the worst and the best-case payoff. It is well known that a weighted average of two quasiconcave functions does not need to be quasiconcave.

The following example demonstrates that complete ignorance is not necessary for a finite population evolutionary stable preference in the class of games considered by Proposition 9 .

Example 3 (Public goods game with multiple Nash equilibria) Consider two players, $i=1,2$, and symmetric payoff functions $\pi\left(a_{i}, a_{i-3}\right)=\frac{1}{4}\left(a_{i}+a_{3-i}\right)^{2}-\frac{1}{2} a_{i}$ with actions $a_{i} \in[0,1]$. This game resembles a public goods game with increasing returns to contribution. Clearly this game has increasing differences and positive aggregate externalities. Since the benefit function $\frac{1}{4}\left(a_{1}+a_{2}\right)^{2}$ is convex in contributions, the only symmetric combinations of actions corresponding to a pure Nash equilibrium are $(0,0)$ and $(1,1)$. Thus Assumption 3 holds. It can be verified that $a_{i}=0$ for $i=1,2$ is the unique fESS. By the arguments in the proof of Proposition 9, it is an ATS (see also Alós-Ferrer and Ania, 2005). The corresponding ATS profile equals to an EKU with complete ignorance and extreme pessimism. Since this equilibrium involves complete ignorance, a non-mutant can not be made worse off by any mutant because $a_{i}=0$ is an fESS and she does not react to a mutant. However, there is a whole range of parameters $\left(\delta_{i}, \alpha_{i}\right)$ for which the best response is $a_{i}=0$ no matter which action a mutant may choose. These parameters are characterized by $\alpha_{i} \in\left[0, \frac{1}{2}\right]$ and $\delta_{i} \in\left[\frac{1}{2\left(1-\alpha_{i}\right)}, 1\right]$. Hence complete ignorance is not necessary for a finite population evolutionary stable preference.

The next example demonstrates what happens if Assumptions 2 and 3 do not hold. It also demonstrates that a fESP may not exist within the class of CEU preferences. In particular, if the game is supermodular and has a unique equilibrium, then there may not exist a finite population evolutionary stable CEU preference.

Example 4 (Public goods game with a dominant action) Consider two players, $i=1,2$, and symmetric payoff functions $\pi\left(a_{i}, a_{3-i}\right)=\left(a_{i}+a_{3-i}\right)^{2}-\epsilon a_{i}$ with actions $a_{i} \in[\epsilon, 1]$ and $0<\epsilon<1$. This game is a variant of the previous example. However, it possesses a unique strict dominant action $a_{i}=1$. Note that Knightian uncertainty respects this dominance, i.e., if an action is strictly dominated, then it is never an equilibrium action under Knightian uncertainty. Thus any symmetric equilibrium under Knightian uncertainty must be $(1,1)$ no matter which degree of optimism and degree of ignorance. However, $(1,1)$ is not fESS since an opponent can make a player relatively worse off than herself by deviating to action $\epsilon$. Hence, there is no finite population evolutionary stable preference within the class of CEU preferences. 
It is straightforward to establish that $a_{i}=\epsilon$ is the unique fESS, and thus by arguments in the proof of Proposition 9, $\epsilon$ is an ATS (see also Alós-Ferrer and Ania, 2005). This example violates Assumption 2 since there exists no profile of preferences within the class of CEU such that the EKU resulting from this preference equals to the ATS profile. It also violates Assumption 3 since $\pi_{i}\left(a_{i}, \inf A_{-i}\right)$ is strictly increasing in $a_{i} \in A$ and not decreasing. From a more general point of view, the example also demonstrates that there is no apparent connection between the unique and strict dominant Nash equilibrium and fESS (as well as no apparent connection between efficiency and fESS).

\section{Concluding Discussion}

We show that with some qualifications, optimistic complete ignorance is evolutionary stable in finite aggregative submodular games and pessimistic complete ignorance is evolutionary stable in finite aggregative supermodular games. Complete ignorance lets players ignore competitors and confers commitment power. The optimistic or pessimistic attitude translates into more aggressive or timid behavior, respectively. Our observations suggest that ambiguity attitudes and the degree of ambiguity may be context specific rather than constant features across all situations a decision maker may face. Our observations also suggest that it is possible to select by evolutionary arguments particular ambiguity attitudes and degrees of ambiguity although such selection is context specific. On the downside, the selection of parameters by evolutionary arguments relies heavily on the clear comparative statics w.r.t. ambiguity attitudes afforded by the particular classes of games and the particular parametric version of Choquet expected utility that we consider in this paper. It is far from clear how to generalize the results.

We conclude with a number of comments:

a. Evolutionary Stable Preferences and Evolutionary Drift: It is immediate that given the abstract set of preferences, the inequalities defining (robust globally) evolutionary stable preferences may not hold strictly. There may be many other preferences that achieve the same fitness. Hence, our notion of (robust globally) evolutionary stable preference does not preclude evolutionary drift among preferences achieving the same fitness. In this sense, our notion of finite population evolutionary stable preferences is a much closer analogy to the notion of neutrally stable strategies than to the standard notion of evolutionary stable strategies. However, any preference taking over the population by evolutionary drift would be behaviorally indistinguishable from the behavior of a homogenous population with the finite population evolutionary stable preference singled out in our results because otherwise it could be invaded by the preferences singled out in our results. To prevent evolutionary drift, further assumptions on the collection of preferences would be required.

Our notion of finite population evolutionary stability is based on the assumption that each member of the relevant population interacts with each other member ("playing-the-field"). In environments with a large population, in which a subset of players is randomly matched to play a game, our conclusions may not obtain if the standard notion of evolutionary stable strategies is applied.

b. Strategic Advantage - An Interpretation as Contest: Instead on focusing on the evolutionary interpretation of the results, the present study may be interpreted as analyzing contests or tournaments among players in which one player may have a different preference than others. 
A player with a finite population evolutionary stable preference maximizes relative payoffs, i.e., $t$ is a fESP of the game $G=\langle N, A, \pi\rangle$ if and only if (recall that we denote by $j$ the mutant and by $i$ a non-mutant)

$$
t \in \arg \max _{t_{j}^{\prime} \in T}\left\{\pi\left(a_{j}^{*}\left(t_{j}^{\prime}, t_{-j}\right), \aleph^{n}\left(\mathbf{a}^{*}\left(t_{j}^{\prime}, t_{-j}\right)\right)\right)-\pi\left(a_{i}^{*}\left(t_{j}^{\prime}, t_{-j}\right), \aleph^{n}\left(\mathbf{a}^{*}\left(t_{j}^{\prime}, t_{-j}\right)\right)\right)\right\} .
$$

A fESP maximizes relative payoffs, a player adopting a fESP may decrease her own material payoffs in equilibrium but decreases material equilibrium payoffs of others even more.

Consider now two preferences $t$ and $t^{\prime}$. Partition the set of players $N$ into two sets $N_{t}$ and $N_{t^{\prime}}$. All players in $N_{t}$ have preference $t$ and analogously for $N_{t^{\prime}}$. Preference $t$ yields a strategic advantage over preference $t^{\prime}$ if at each equilibrium $\mathbf{a}^{*} \in \mathbf{A}$ of the game $G=\left\langle N, A,\left(t_{i}\right)_{i \in N_{t}},\left(t_{j}^{\prime}\right)_{j \in N_{t^{\prime}}}\right\rangle$ we have

$$
\pi_{i}\left(\mathbf{a}^{*}\right) \geq \pi_{j}\left(\mathbf{a}^{*}\right) \text { for all }(i, j) \in N_{t} \times N_{t^{\prime}}
$$

with strict inequality for some $(i, j) \in N_{t} \times N_{t^{\prime}}$ (see Koçkesen, Ok, and Sethi, 2000a, b). In our case, a preference yielding a strategic advantage is a strict robust $\left|N_{t^{\prime}}\right|$ evolutionary stable preference (that would exclude evolutionary drift as discussed above). Suppose we restrict $T_{G}$ suitably in a non-trivial way such to exclude the possibility of evolutionary drift. E.g., let $T_{G}$ consist just of expected utility preferences with Bernoulli utility $\pi$ and the Choquet expected utility with neo-additive capacity as singled out in our results. Then our result on symmetric aggregative submodular games with aggregate externalities implies that optimism and complete ignorance yields a strategic advantage over other preferences in the set $T_{G}$. Similarly, if $N_{t^{\prime}}$ is a singleton, then our result on symmetric aggregative supermodular games with aggregate externalities implies that extreme pessimism and complete ignorance yields a strategic advantage over other preferences in the set $T_{G}$.

c. Behavioral indistinguishability of Choquet expected utility and relative payoff maximization: It is well-known from Schaffer $(1988,1989)$ that finite population evolutionary stable strategy captures the notion of relative payoff maximization. Thus, another way of interpreting our results is that they show that in some important classes of games, Choquet expected utility with respect to some particular neo-additive capacities is behaviorally indistinguishable from relative payoff maximization. For instance, in aggregate submodular games with negative aggregative externalities (e.g., Example 1), players can be made suitably aggressive by increasing the degree of optimism in Choquet expected utility with respect to neo-additive capacities. Such aggression is required for relative payoff maximization. As it is well-known from a number of results in the literature (Vega-Redondo, 1997, Schipper, 2003, Alós-Ferrer and Ania, 2005, Schipper, 2009, Duersch, Oechssler, and Schipper, 2012) that such aggression may decrease own payoffs but decreases payoffs of others even more in such games.

d. Observability of Preferences: It has been noted in the literature on the evolution of preferences that the commitment effect of preferences vanishes if preferences are not perfectly observable (e.g., Samuelson, 2001, Ok and Vega-Redondo, 2001, Ely and Yilankaya, 2001, Dekel, Ely, and Yilankaya, 2007). We believe that this critique does not apply to our setting because we show that a preference with complete ignorance is finite population evolutionary stable. Thus, an action by player with this evolutionary stable preference does not depend on the observability of the opponents' actions or preferences. With a different approach, Heifetz, Shannon, and Spiegel (2007a) also show that payoff maximizing behavior may not need to prevail even if preferences are imperfectly observable. 
e. Evolutionary Dynamics: So far, we just considered a static concept of evolutionary stability and were silent on any dynamic process of preference evolution. Only few authors considered explicitly the dynamics of preference evolution (Huck, Kirchsteiger, and Oechssler, 2005, Sandholm, 2001, Heifetz, Shannon, and Spiegel, 2007b, and Possajennikov, 2005). A dynamic analysis in our setting should consider beside the preference dynamics also a faster learning process for equilibrium under Knightian uncertainty. Unfortunately, no learning process for equilibrium under Knightian uncertainty has been proposed. Leaving this conceptional issue aside, we can propose a process of preference adaption based on imitate-the-best. In reality, testimonies of (un-)successful people educate us in some situations on "think positive" or "be careful" even though this education may not be conscious. Hence it is not unreasonable to assume that attitudes towards uncertainty may be imitated. Consider the following model of imitation à la Vega-Redondo (1997): In each period every player has a strict positive probability bounded away from one to adjust her preference. If a player adjusts, then she mimics the preference of the most successful player in the previous round (note that in contrast to above discussion this imitation process requires that success and preferences are observable). For simplicity consider a finite set of preferences that at least entails complete ignorance and "relevant" degrees of optimism. The imitation dynamics induces a discrete time finite Markov chain on the space of preference profiles. Focusing on intra-group symmetric equilibria it can be shown that the set of absorbing sets includes each identical preference profile. If we assume that each player may make mistakes in imitating preferences (noise), i.e., with a small probability she selects any preference profile when adjusting her preference, then the resulting perturbed Markov chain is ergodic and irreducible. We can now focus on the unique limiting invariant distribution of preferences when the noise goes to zero. This is the long run distribution interpreted as the average proportion of time a player selects each preference. For aggregative strict submodular games with aggregate externalities we conjecture based on results by Schipper (2003) and arguments in the proof of Proposition 8, that the evolutionary stable preference with complete ignorance and optimism is in the support of the long run distribution, i.e., "stochastically stable". Similarly, for aggregative strict supermodular games with aggregate externalities, we conjecture that the evolutionary stable preference with complete ignorance and extreme pessimism is in the support of the long run distribution.

f. Short Run "Industry" Dynamics and Entry: While evolutionary stability focuses on long run outcomes, in the short run interesting dynamics of profits can arise. Consider a standard Cournot oligopoly and assume that all firms choose individually optimal according to the evolutionary stable preference which involves complete ignorance and optimism. Suppose now that there is a mutant who is a realist. A realist improves her profit compared to her premutant profit before because she plays a best response to the opponents' quantities. However, she raises the profits of the opponents' even more. Before the realistic mutant is driven out in the medium run, all may earn higher profits, which may attract entry by additional firms.

g. Strategic Delegation and Optimism: Eichberger, Kelsey, and Schipper (2009) show among others that a principal prefers to delegate to an optimistic manager in both Cournot and Bertrand oligopoly. This is surprising since results are usually reversed when one goes from standard Cournot to standard Bertrand oligopoly. The reason for the results is that an optimistic manager in Cournot oligopoly is more aggressive and less expensive, while she is less aggressive and less expensive in Bertrand oligopoly. From our analysis in this article it is clear that a principal delegating to an optimistic manager in a supermodular Bertrand oligopoly may not survive (unless costs of managers are the main factor entering profits). Since the manager is 
less aggressive, the firm may make less material payoffs then a competitor with more pessimistic manager. If potential investors cannot observe whether firms maximize profits but only which firm has higher profits and allocate investment funds to firms with profits higher than others, then firms with principals delegating to an optimistic manager may not survive. This is in contrast to Cournot oligopoly, in which firms with principals delegating to optimistic managers survive.

\section{A Proofs}

\section{Proof of Lemma 1}

If $A_{i}$ is a subcomplete sublattice of a complete lattice for all players $i \in N$, then it is a complete lattice. The direct product $A_{-i}=\times_{j \in N \backslash\{i\}} A_{j}$ of complete lattices is a complete lattice. By the Frink-Birkhoff theorem, a lattice is complete if and only if it is compact in its interval-topology (Topkis, 1998, pp. 29). Since $\pi_{i}$ is continuous in $a_{-i}$ on $A_{-i}$ for every $a_{i}$, it follows from Weierstrass' theorem that it attains minima and maxima w.r.t. $a_{-i} \in A_{-i}$ for every $a_{i} \in A_{i}$. Thus, both $\arg \max _{a_{-i} \in A_{-i}} \pi_{i}\left(a_{i}, a_{-i}\right)$ and $\arg \min _{a_{-i} \in A_{-i}} \pi_{i}\left(a_{i}, a_{-i}\right)$ are nonempty for every $a_{i} \in A_{i}$. Since $A_{-i}$ is a complete lattice, it has a greatest and least element in $A_{-i}$ which are $\sup _{A_{-i}} A_{-i}$ and $\inf _{A_{-i}} A_{-i}$, respectively. The remainder of the proof now follows directly from the definitions of positive and negative externalities, respectively.

\section{Proof of Lemma 2}

We prove a more general ordinal version of Lemma 2 that might be interesting on its own right. To this end, we introduce some definitions. A real valued function $f$ on a partially ordered set $X \times T$ satisfies the (dual) single crossing property in $(x, t)$ on $X \times T$ if for all $x^{\prime \prime} \triangleright x^{\prime}$ and $t^{\prime \prime} \triangleright t^{\prime}$,

$$
\begin{aligned}
& f\left(x^{\prime \prime}, t^{\prime}\right) \geq f\left(x^{\prime}, t^{\prime}\right) \quad \Rightarrow(\Leftarrow) \quad f\left(x^{\prime \prime}, t^{\prime \prime}\right) \geq f\left(x^{\prime}, t^{\prime \prime}\right), \\
& f\left(x^{\prime \prime}, t^{\prime}\right)>f\left(x^{\prime}, t^{\prime}\right) \Rightarrow(\Leftarrow) \quad f\left(x^{\prime \prime}, t^{\prime \prime}\right)>f\left(x^{\prime}, t^{\prime \prime}\right) .
\end{aligned}
$$

We say that $f$ satisfies the strict (dual) single crossing property in $(x, t)$ on $X \times T$ if for all $x^{\prime \prime} \triangleright x^{\prime}$ and $t^{\prime \prime} \triangleright t^{\prime}$

$$
f\left(x^{\prime \prime}, t^{\prime}\right) \geq(>) f\left(x^{\prime}, t^{\prime}\right) \quad \Rightarrow(\Leftarrow) \quad f\left(x^{\prime \prime}, t^{\prime \prime}\right)>(\geq) f\left(x^{\prime}, t^{\prime \prime}\right) .
$$

It is straight-forward to verify that increasing (decreasing) differences implies the (dual) single crossing property but not vice versa. The same holds for the strict versions.

Lemma 5 Let $G=\left\langle N,\left(A_{i}\right),\left(\pi_{i}\right)\right\rangle$ be an aggregative strategic game with $A_{i}$ being a chain for each $i \in N$, and let $\mathbf{a}^{\circ}$ be a symmetric ATS profile and $\mathbf{a}^{*}$ be a symmetric Nash equilibrium of $G$. If the aggregative game $G$ is such that $\pi_{i}$ satisfies

(i) the dual single crossing property in $\left(a_{i}, \aleph^{n}(\mathbf{a})\right)$ on $A_{i} \times X$ and $G$ has strict negative (resp. positive) aggregate externalities, or

(ii) the strict dual single crossing property in $\left(a_{i}, \aleph^{n}(\mathbf{a})\right)$ on $A_{i} \times X$ and $G$ has negative (resp. positive) aggregate externalities,

then $\mathbf{a}^{\circ} \unrhd \mathbf{a}^{*}\left(\right.$ resp. $\left.\mathbf{a}^{\circ} \unlhd \mathbf{a}^{*}\right)$.

Proof. Since $\mathbf{a}^{\circ}$ is an ATS and $\mathbf{a}^{*}$ is a Nash equilibrium of $G$, we have by definition

$$
\begin{aligned}
& \pi_{i}\left(a_{i}^{\circ}, \aleph^{n}\left(a_{i}^{\circ}, a_{-i}^{\circ}\right)\right) \geq \pi_{i}\left(a_{i}^{*}, \aleph^{n}\left(a_{i}^{\circ}, a_{-i}^{\circ}\right)\right), \\
& \pi_{i}\left(a_{i}^{*}, \aleph^{n}\left(a_{i}^{*}, a_{-i}^{*}\right)\right) \geq \pi_{i}\left(a_{i}^{\circ}, \aleph^{n}\left(a_{i}^{\circ}, a_{-i}^{*}\right)\right),
\end{aligned}
$$


for all $i \in N$. Consider case (i), and let $G$ have strict negative aggregate externalities and $A_{i}$ be a chain. Suppose to the contrary that for $i \in N$ we have $a_{i}^{\circ} \triangleleft a_{i}^{*}$. By the dual single crossing property of $\pi_{i}$ in $\left(a_{i}, \aleph(\mathbf{a})\right)$ on $A_{i} \times X$,

$$
\pi_{i}\left(a_{i}^{*}, \aleph^{n}\left(a_{i}^{*}, a_{-i}^{*}\right)\right) \geq \pi_{i}\left(a_{i}^{\circ}, \aleph^{n}\left(a_{i}^{\circ}, a_{-i}^{*}\right)\right) \Rightarrow \pi_{i}\left(a_{i}^{*}, \aleph^{n}\left(a_{i}^{*}, a_{-i}^{\circ}\right)\right) \geq \pi_{i}\left(a_{i}^{\circ}, \aleph^{n}\left(a_{i}^{\circ}, a_{-i}^{\circ}\right)\right) .
$$

Since $G$ has strict negative aggregate externalities,

$$
\pi_{i}\left(a_{i}^{*}, \aleph^{n}\left(a_{i}^{*}, a_{-i}^{\circ}\right)\right) \geq \pi_{i}\left(a_{i}^{\circ}, \aleph^{n}\left(a_{i}^{\circ}, a_{-i}^{\circ}\right)\right) \Rightarrow \pi_{i}\left(a_{i}^{*}, \aleph^{n}\left(a_{i}^{\circ}, a_{-i}^{\circ}\right)\right)>\pi_{i}\left(a_{i}^{\circ}, \aleph^{n}\left(a_{i}^{\circ}, a_{-i}^{\circ}\right)\right),
$$

which is a contradiction to inequality (13). The case for strict positive externalities follows analogously.

Now consider case (ii). Again, suppose to the contrary that for $i \in N$ we have $a_{i}^{\circ} \triangleleft a_{i}^{*}$. By the strict dual single crossing property of $\pi_{i}$ in $\left(a_{i}, \aleph(\mathbf{a})\right)$ on $A_{i} \times X$,

$$
\pi_{i}\left(a_{i}^{*}, \aleph^{n}\left(a_{i}^{*}, a_{-i}^{*}\right)\right) \geq \pi_{i}\left(a_{i}^{\circ}, \aleph^{n}\left(a_{i}^{\circ}, a_{-i}^{*}\right)\right) \Rightarrow \pi_{i}\left(a_{i}^{*}, \aleph^{n}\left(a_{i}^{*}, a_{-i}^{\circ}\right)\right)>\pi_{i}\left(a_{i}^{\circ}, \aleph^{n}\left(a_{i}^{\circ}, a_{-i}^{\circ}\right)\right) .
$$

Since $G$ has negative aggregate externalities,

$$
\pi_{i}\left(a_{i}^{*}, \aleph^{n}\left(a_{i}^{*}, a_{-i}^{\circ}\right)\right)>\pi_{i}\left(a_{i}^{\circ}, \aleph^{n}\left(a_{i}^{\circ}, a_{-i}^{\circ}\right)\right) \Rightarrow \pi_{i}\left(a_{i}^{*}, \aleph^{n}\left(a_{i}^{\circ}, a_{-i}^{\circ}\right)\right)>\pi_{i}\left(a_{i}^{\circ}, \aleph^{n}\left(a_{i}^{\circ}, a_{-i}^{\circ}\right)\right),
$$

which is a contradiction to inequality (13). The case for positive externalities follows analogously.

Lemma 2 is now an immediate corollary of Lemma 5.

\section{Proof of Lemma 3}

We need to show that if $\pi_{i}$ is supermodular in $a_{i}$ on $A_{i}$ and has increasing (resp. decreasing) differences in $\left(a_{i}, a_{-i}\right)$ on $\mathbf{A}$ then $u_{i}\left(\alpha_{i}, \delta_{i}\right)$ is supermodular in $a_{i}$ on $A_{i}$ and has increasing (resp. decreasing) differences in $\left(a_{i}, a_{-i}\right)$ on $\mathbf{A}$ for each $\left(\alpha_{i}, \delta_{i}\right) \in[0,1]^{2}$. If $\pi_{i}$ is supermodular in $a_{i}$ on $A_{i}$ and has increasing (resp. decreasing) differences in $\left(a_{i}, a_{-i}\right)$ on $\mathbf{A}$, then for any scalar $\gamma \geq 0$ also $\gamma \pi_{i}$ is supermodular in $a_{i}$ on $A_{i}$ and has increasing (resp. decreasing) differences in $\left(a_{i}, a_{-i}\right)$ on $\mathbf{A}$ (Topkis, 1998, Lemma 2.6.1. (a)). $\nabla_{i}$ and $\boldsymbol{w}_{i}$ are both supermodular in $a_{i}$ on $A_{i}$ by definition and constant in $a_{-i}$ on $A_{-i}$. Since $u_{i}$ is for each $\left(\alpha_{i}, \delta_{i}\right) \in[0,1]^{2}$ a sum of supermodular functions in $a_{i}$ on $A_{i}$ having increasing (resp. decreasing) differences in $\left(a_{i}, a_{-i}\right)$ on $\mathbf{A}$, it is supermodular in $a_{i}$ on $A_{i}$ and has increasing (resp. decreasing) differences $\left(a_{i}, a_{-i}\right)$ on A (Topkis, 1998, Lemma 2.6.1. (b)). By analogous arguments, the result extends to the strict versions (see Topkis, 1998, p. 49).

\section{Proof of Lemma 4}

By Lemma 3, if $G$ is supermodular (resp. submodular), then $G(\boldsymbol{\alpha}, \boldsymbol{\delta})$ is supermodular (resp. submodular) for each $(\boldsymbol{\alpha}, \boldsymbol{\delta}) \in[0,1]^{n} \times[0,1]^{n}$. Thus $u_{i}$ is supermodular in $a_{i}$ on $A_{i}$ and has increasing (resp. decreasing) differences in $\left(a_{i}, a_{-i}\right)$ on A. Since $u_{i}$ is supermodular in $a_{i}$ on $A_{i}, b_{i}\left(a_{-i}, \alpha_{i}, \delta_{i}\right)$ is a sublattice of $A_{i}$ for each $a_{-i} \in A_{-i}$ and $\left(\alpha_{i}, \delta_{i}\right) \in[0,1]^{2}$ by Topkis (1998), Theorem 2.7.1. Since $u_{i}$ has increasing (resp. decreasing) differences in $\left(a_{i}, a_{-i}\right)$ on $\mathbf{A}, b_{i}\left(a_{-i}, \alpha_{i}, \delta_{i}\right)$ is increasing (resp. decreasing) in $a_{-i}$ on $\left\{a_{-i} \in A_{-i}: b_{i}\left(a_{-i}, \alpha_{i}, \delta_{i}\right) \neq \emptyset\right\}$ for any $\left(\alpha_{i}, \delta_{i}\right) \in[0,1]^{2}$ by Topkis (1998), Theorem 2.8.1.

\section{Proof of Proposition 1}

If $\pi_{i}$ is continuous on $A_{-i}$ for every $a_{i} \in A_{i}$, then $u_{i}$ is well-defined (Remark 1 ). If $\pi_{i}$ is upper semicontinuous on $A_{i}$, then $u_{i}$ is upper semicontinuous on $A_{i}$ since limits are preserved under algebraic operations. The result follows now from Lemmata 3 and 4 and Zhou's (1994) generalization of Tarski's fixed point 
theorem.

Note that in Proposition 1 we do not claim that the set of equilibria under Knightian uncertainty is a sublattice of $\mathbf{A}$. Thus if $\mathbf{a}=\left(a_{1}, \ldots, a_{n}\right)$ and $\mathbf{a}^{\prime}=\left(a_{1}^{\prime}, \ldots, a_{n}^{\prime}\right)$ are both equilibria under Knightian uncertainty for $G(\boldsymbol{\alpha}, \boldsymbol{\delta})$, then $\left(a_{1} \vee a_{1}^{\prime}, \ldots, a_{n} \vee a_{n}^{\prime}\right)$ and $\left(a_{1} \wedge a_{1}^{\prime}, \ldots, a_{n} \wedge a_{n}^{\prime}\right)$ may not be equilibria under Knightian uncertainty for the game $G(\boldsymbol{\alpha}, \boldsymbol{\delta})$ (see for an example Zhou (1994), p. 299).

Remark 3 In addition to the assumptions of Proposition 1 assume that $n=2$ and that $A_{i}$ is a chain for $i \in\{1,2\}$. Then then for any $(\boldsymbol{\alpha}, \boldsymbol{\delta}) \in[0,1]^{n} \times[0,1]^{n}$ the set of equilibria under Knightian uncertainty is a subcomplete sublattice and a greatest and least equilibrium exist.

Proof of Remark. This follows from Proposition 1 and a result by Echenique (2003) who observed that the set of Nash equilibria forms a sublattice in two-player games with totally ordered action sets for which each player's best response correspondence is increasing in the strong set order.

\section{Proof of Proposition 2}

Since $\pi_{i}$ is defined on $A_{i}$ and the range of sums of opponents actions and continuous in latter, by arguments similar to the ones for proving Remark $1, G(\boldsymbol{\alpha}, \boldsymbol{\delta})$ is well-defined for all $(\boldsymbol{\alpha}, \boldsymbol{\delta}) \in[0,1]^{n} \times[0,1]^{n}$. Submodularity of $G$ implies by Lemma 3 submodularity of $G(\boldsymbol{\alpha}, \boldsymbol{\delta})$ for all $(\boldsymbol{\alpha}, \boldsymbol{\delta}) \in[0,1]^{n} \times[0,1]^{n}$. Thus for all $i \in N, u_{i}\left(\alpha_{i}, \delta_{i}\right)$ has decreasing differences in $\left(a_{i}, \sum_{j \in N \backslash\{i\}} a_{j}\right)$. By Lemma $4, b_{i}\left(\alpha_{i}, \delta_{i}\right)$ is decreasing in $\sum_{j \in N \backslash\{i\}} a_{j}$. Since $\pi_{i}$ is continuous in both variables, $u_{i}\left(\alpha_{i}, \delta_{i}\right)$ is continuous in $a_{i}$ and $\sum_{j \in N \backslash\{j\}} a_{j}$ because limits are preserved under algebraic operations. Thus $b_{i}\left(\sum_{j \in N \backslash\{i\}} a_{j}, \alpha_{i}, \delta_{i}\right)$ is non-empty for any $\sum_{j \in N \backslash\{i\}} a_{j}$. It also implies that $b_{i}\left(\alpha_{i}, \delta_{i}\right)$ is upper-hemicontinuous in $\sum_{j \in N \backslash\{i\}} a_{j}$. Thus the conditions are sufficient for a theorem by Kukushkin (1994) by which there exists a Nash equilibrium in pure actions of the game $G(\boldsymbol{\alpha}, \boldsymbol{\delta})$.

\section{Proof of Proposition 3}

By Remark $2, b_{i}\left(a_{-i}, \alpha_{i}, 1\right)$ is constant and therefore trivially increasing in $a_{-i}$ on $A_{-i}$ for any $\alpha_{i} \in[0,1]$ for all $i \in N$. Hence the result follows as a special case from the proof of Proposition 1.

\section{Proof of Proposition 4}

For an equilibrium under Knightian uncertainty to exist for $(\boldsymbol{\alpha}, \mathbf{1}), u_{i}\left(\alpha_{i}, 1\right)$ must be well-defined. If $\pi_{i}$ is strictly concave in $a_{i}$, then for any $\alpha_{i} \in[0,1], u_{i}\left(\alpha_{i}, 1\right)$ is strictly concave in $a_{i}$ since it is a weighted sum of strictly concave functions. Strict concavity of $u_{i}\left(a_{i}, 1\right)$ is sufficient for $b_{i}\left(a_{-i}, \alpha_{i}, 1\right)$ being unique for all $a_{-i} \in A_{-i}$. By Remark 2, if $\delta_{i}=1$ then $b_{i}\left(a_{-i}, \alpha_{i}, 1\right)$ is constant for all $a_{-i} \in A_{-i}$. Hence, if there exists an equilibrium under Knightian uncertainty with $\delta_{i}=1$ for all $i \in N$, then it must be unique with each player choosing her strictly dominant action.

\section{Proof of Proposition 5}

Proposition 5 is a direct corollary of the following two lemmata:

Lemma 6 If $\pi_{i}$ has

- increasing differences in $\left(a_{i}, a_{-i}\right)$ on $\mathbf{A}$ and positive (resp. negative) externalities, or 
- decreasing differences in $\left(a_{i}, a_{-i}\right)$ on $\mathbf{A}$ and negative (resp. positive) externalities,

then $u_{i}$ has increasing (resp. decreasing) differences in $\left(a_{i}, \alpha_{i}\right)$ on $A_{i} \times[0,1]$ for all $a_{-i} \in A_{-i}$ and $\delta_{i} \in[0,1]$. The result extends to the strict versions.

Proof of Lemma. If $\pi_{i}$ has decreasing (increasing) differences in $\left(a_{i}, a_{-i}\right)$ on $\mathbf{A}$ then for all $a_{i}^{\prime \prime} \triangleright a_{i}^{\prime}$,

$$
\pi_{i}\left(a_{i}^{\prime \prime}, \sup _{\mathbf{A}} A_{-i}\right)-\pi_{i}\left(a_{i}^{\prime}, \sup _{\mathbf{A}} A_{-i}\right) \leq(\geq) \pi_{i}\left(a_{i}^{\prime \prime}, \inf _{\mathbf{A}} A_{-i}\right)-\pi_{i}\left(a_{i}^{\prime}, \inf _{\mathbf{A}} A_{-i}\right) .
$$

By Remark 1 it follows that if $\pi_{i}$ has [decreasing differences in $\left(a_{i}, a_{-i}\right)$ on $\mathbf{A}$ and negative (resp. positive) externalities] or [increasing differences in $\left(a_{i}, a_{-i}\right)$ on $\mathbf{A}$ and positive (resp. negative) externalities], then for all $a_{i}^{\prime \prime} \triangleright a_{i}^{\prime}$,

$$
\mathbf{W}_{i}\left(a_{i}^{\prime \prime}\right)-\mathbf{W}_{i}\left(a_{i}^{\prime}\right) \leq(\geq) \oslash_{i}\left(a_{i}^{\prime \prime}\right)-\oslash_{i}\left(a_{i}^{\prime}\right)
$$

Let $\alpha_{i}^{\prime}, \alpha_{i}^{\prime \prime} \in[0,1]$ with $\alpha_{i}^{\prime \prime} \geq \alpha_{i}^{\prime}$. It follows from the last inequality that

$$
\begin{gathered}
\alpha_{i}^{\prime \prime}\left[\left(\bigcirc_{i}\left(a_{i}^{\prime \prime}\right)-\bigcirc_{i}\left(a_{i}^{\prime}\right)\right)-\left(\boldsymbol{\Psi}_{i}\left(a_{i}^{\prime \prime}\right)-\Psi_{i}\left(a_{i}^{\prime}\right)\right)\right] \geq(\leq) \\
\alpha_{i}^{\prime}\left[\left(\bigcirc_{i}\left(a_{i}^{\prime \prime}\right)-\bigcirc_{i}\left(a_{i}^{\prime}\right)\right)-\left(\boldsymbol{\Psi}_{i}\left(a_{i}^{\prime \prime}\right)-\boldsymbol{\Psi}_{i}\left(a_{i}^{\prime}\right)\right)\right] .
\end{gathered}
$$

This is equivalent to

$$
\begin{aligned}
& \alpha_{i}^{\prime \prime}\left[\bigcirc_{i}\left(a_{i}^{\prime \prime}\right)-\bigcirc_{i}\left(a_{i}^{\prime}\right)\right]-\alpha_{i}^{\prime \prime}\left[\mathbf{W}_{i}\left(a_{i}^{\prime \prime}\right)-\mathbf{w}_{i}\left(a_{i}^{\prime}\right)\right] \geq(\leq) \\
& \alpha_{i}^{\prime}\left[\bigcirc_{i}\left(a_{i}^{\prime \prime}\right)-\bigcirc_{i}\left(a_{i}^{\prime}\right)\right]-\alpha_{i}^{\prime}\left[\mathbf{W}_{i}\left(a_{i}^{\prime \prime}\right)-\mathbf{W}_{i}\left(a_{i}^{\prime}\right)\right] \\
& \alpha_{i}^{\prime \prime}\left[\bigcirc_{i}\left(a_{i}^{\prime \prime}\right)-\bigcirc_{i}\left(a_{i}^{\prime}\right)\right]+\left[\mathbf{\Psi}_{i}\left(a_{i}^{\prime \prime}\right)-\boldsymbol{\Psi}_{i}\left(a_{i}^{\prime}\right)\right]-\alpha_{i}^{\prime \prime}\left[\boldsymbol{\Psi}_{i}\left(a_{i}^{\prime \prime}\right)-\boldsymbol{\Psi}_{i}\left(a_{i}^{\prime}\right)\right] \geq(\leq) \\
& \alpha_{i}^{\prime}\left[\bigcirc_{i}\left(a_{i}^{\prime \prime}\right)-\bigcirc_{i}\left(a_{i}^{\prime}\right)\right]+\left[\mathbf{\Psi}_{i}\left(a_{i}^{\prime \prime}\right)-\mathbf{W}_{i}\left(a_{i}^{\prime}\right)\right]-\alpha_{i}^{\prime}\left[\mathbf{\Psi}_{i}\left(a_{i}^{\prime \prime}\right)-\mathbf{\Psi}_{i}\left(a_{i}^{\prime}\right)\right] \\
& \alpha_{i}^{\prime \prime}\left[\bigcirc_{i}\left(a_{i}^{\prime \prime}\right)-\bigcirc_{i}\left(a_{i}^{\prime}\right)\right]+\left(1-\alpha_{i}^{\prime \prime}\right)\left[\mathbf{\Psi}_{i}\left(a_{i}^{\prime \prime}\right)-\mathbf{\Psi}_{i}\left(a_{i}^{\prime}\right)\right] \geq(\leq) \\
& \alpha_{i}^{\prime}\left[\bigcirc_{i}\left(a_{i}^{\prime \prime}\right)-\bigcirc_{i}\left(a_{i}^{\prime}\right)\right]+\left(1-\alpha_{i}^{\prime}\right)\left[\mathbf{\Psi}_{i}\left(a_{i}^{\prime \prime}\right)-\boldsymbol{\Psi}_{i}\left(a_{i}^{\prime}\right)\right] .
\end{aligned}
$$

Consider any $\delta_{i} \in[0,1]$. Then the previous inequality implies

$$
\begin{gathered}
\alpha_{i}^{\prime \prime} \delta_{i}\left[\bigcirc_{i}\left(a_{i}^{\prime \prime}\right)-\bigcirc_{i}\left(a_{i}^{\prime}\right)\right]+\left(1-\alpha_{i}^{\prime \prime}\right) \delta_{i}\left[\boldsymbol{\Psi}_{i}\left(a_{i}^{\prime \prime}\right)-\boldsymbol{\Psi}_{i}\left(a_{i}^{\prime}\right)\right] \geq(\leq) \\
\alpha_{i}^{\prime} \delta_{i}\left[\bigcirc_{i}\left(a_{i}^{\prime \prime}\right)-\bigcirc_{i}\left(a_{i}^{\prime}\right)\right]+\left(1-\alpha_{i}^{\prime}\right) \delta_{i}\left[\mathbf{W}_{i}\left(a_{i}^{\prime \prime}\right)-\boldsymbol{\Psi}_{i}\left(a_{i}^{\prime}\right)\right],
\end{gathered}
$$

which in turn implies

$$
\begin{aligned}
& \alpha_{i}^{\prime \prime} \delta_{i}\left[\bigcirc_{i}\left(a_{i}^{\prime \prime}\right)-\bigcirc_{i}\left(a_{i}^{\prime}\right)\right]+\left(1-\alpha_{i}^{\prime \prime}\right) \delta_{i}\left[\mathbf{W}_{i}\left(a_{i}^{\prime \prime}\right)-\boldsymbol{\Psi}_{i}\left(a_{i}^{\prime}\right)\right]+ \\
& \left(1-\delta_{i}\right)\left[\pi_{i}\left(a_{i}^{\prime \prime}, a_{-i}\right)-\pi_{i}\left(a_{i}^{\prime}, a_{-i}\right)\right] \geq(\leq) \\
& \alpha_{i}^{\prime} \delta_{i}\left[\bigcirc_{i}\left(a_{i}^{\prime \prime}\right)-\bigcirc_{i}\left(a_{i}^{\prime}\right)\right]+\left(1-\alpha_{i}^{\prime}\right) \delta_{i}\left[\boldsymbol{\Psi}_{i}\left(a_{i}^{\prime \prime}\right)-\delta_{i}\left(a_{i}^{\prime}\right)\right]+ \\
& \left(1-\delta_{i}\right)\left[\pi_{i}\left(a_{i}^{\prime \prime}, a_{-i}\right)-\pi_{i}\left(a_{i}^{\prime}, a_{-i}\right)\right] \\
& \delta_{i}\left[\alpha_{i}^{\prime \prime} \oslash_{i}\left(a_{i}^{\prime \prime}\right)+\left(1-\alpha_{i}^{\prime \prime}\right) \boldsymbol{\Psi}_{i}\left(a_{i}^{\prime \prime}\right)\right]+\left(1-\delta_{i}\right) \pi_{i}\left(a_{i}^{\prime \prime}, a_{-i}\right) \\
& -\delta_{i}\left[\alpha_{i}^{\prime \prime} \bigcirc_{i}\left(a_{i}^{\prime}\right)+\left(1-\alpha_{i}^{\prime \prime}\right) \mathbf{w}_{i}\left(a_{i}^{\prime}\right)\right]-\left(1-\delta_{i}\right) \pi_{i}\left(a_{i}^{\prime}, a_{-i}\right) \geq(\leq) \\
& \delta_{i}\left[\alpha_{i}^{\prime} \bigcirc_{i}\left(a_{i}^{\prime \prime}\right)+\left(1-\alpha_{i}^{\prime}\right) \boldsymbol{W}_{i}\left(a_{i}^{\prime \prime}\right)\right]+\left(1-\delta_{i}\right) \pi_{i}\left(a_{i}^{\prime \prime}, a_{-i}\right) \\
& -\delta_{i}\left[\alpha_{i}^{\prime} \bigcirc_{i}\left(a_{i}^{\prime}\right)+\left(1-\alpha_{i}^{\prime}\right) \mathbf{\Psi}_{i}\left(a_{i}^{\prime}\right)\right]-\left(1-\delta_{i}\right) \pi_{i}\left(a_{i}^{\prime}, a_{-i}\right) .
\end{aligned}
$$

Hence we have

$$
u_{i}\left(a_{i}^{\prime \prime}, a_{-i}, \alpha_{i}^{\prime \prime}, \delta_{i}\right)-u_{i}\left(a_{i}^{\prime}, a_{-i}, \alpha_{i}^{\prime \prime}, \delta_{i}\right) \geq(\leq) \quad u_{i}\left(a_{i}^{\prime \prime}, a_{-i}, \alpha_{i}^{\prime}, \delta_{i}\right)-u_{i}\left(a_{i}^{\prime}, a_{-i}, \alpha_{i}^{\prime}, \delta_{i}\right) .
$$

The proof holds analogously for strict versions. 
Lemma 7 (Monotone Optimal Selections) If $u_{i}\left(a_{i}, a_{-i}, \alpha_{i}, \delta_{i}\right)$ is supermodular in $a_{i}$ on $A_{i}$ and has increasing (resp. decreasing) differences in $\left(a_{i}, \alpha_{i}\right)$ on $A_{i} \times[0,1]$ for each $a_{-i} \in A_{-i}$ and $\delta_{i} \in[0,1]$, then $b_{i}\left(a_{-i}, \alpha_{i}, \delta_{i}\right)$ is increasing (resp. decreasing) in $\alpha_{i}$ on $\left\{\alpha_{i} \in[0,1]: b_{i}\left(a_{-i}, \alpha_{i}, \delta_{i}\right) \neq \emptyset\right\}$ for $\delta_{i} \in[0,1]$. If in addition $u_{i}\left(a_{i}, a_{-i}, \alpha_{i}, \delta_{i}\right)$ has strictly increasing (resp. decreasing) differences in $\left(a_{i}, \alpha_{i}\right)$ on $A_{i} \times[0,1]$ for each $a_{-i} \in A_{-i}$ and $\delta_{i} \in[0,1], \alpha_{i}^{\prime \prime}>\alpha_{i}^{\prime}$ in $[0,1]$, and for any $a_{-i} \in A_{-i}, a_{i}^{\prime} \in b_{i}\left(a_{-i}, \alpha_{i}^{\prime}, \delta_{i}\right)$ and $a_{i}^{\prime \prime} \in b_{i}\left(a_{-i}, \alpha_{i}^{\prime \prime}, \delta_{i}\right)$, then $a_{i}^{\prime} \unlhd(\unrhd) a_{i}^{\prime \prime}$. In this case, if one picks any $a_{i}\left(\alpha_{i}\right)$ in $b_{i}\left(a_{-i}, \alpha_{i}, \delta_{i}\right)$ for each $\alpha_{i}$ with $b_{i}\left(a_{-i}, \alpha_{i}, \delta_{i}\right)$ nonempty, then $a_{i}\left(\alpha_{i}\right)$ is increasing (resp. decreasing) in $\alpha_{i}$ on $\left\{\alpha_{i} \in[0,1]\right.$ : $\left.b_{i}\left(a_{-i}, \alpha_{i}, \delta_{i}\right) \neq \emptyset\right\}$.

Proof of Lemma. Pick any $\alpha_{i}^{\prime \prime}$ and $\alpha_{i}^{\prime}$ in $[0,1]$ with $\alpha_{i}^{\prime \prime}>\alpha_{i}^{\prime}$, and for any $\left(a_{-i}, \delta_{i}\right) \in A_{-i} \times[0,1]$, $a_{i}^{\prime} \in b_{i}\left(a_{-i}, \alpha_{i}^{\prime}, \delta_{i}\right)$ and $a_{i}^{\prime \prime} \in b_{i}\left(a_{-i}, \alpha_{i}^{\prime \prime}, \delta_{i}\right)$.

First, consider strictly increasing differences of $u_{i}$ in $\left(a_{i}, \alpha_{i}\right)$, and suppose that it is not true that $a_{i}^{\prime} \unlhd a_{i}^{\prime \prime}$. Then $a_{i}^{\prime \prime} \triangleleft a_{i}^{\prime} \vee a_{i}^{\prime \prime}$ and so using the hypothesis that $u_{i}\left(a_{i}, a_{-i}, \alpha_{i}, \delta_{i}\right)$ is for any $\left(a_{-i}, \delta_{i}\right) \in A_{-i} \times[0,1]$ supermodular in $a_{i}$ and has strictly increasing differences in $\left(a_{i}, \alpha_{i}\right)$,

$$
\begin{aligned}
0 & \leq u_{i}\left(a_{i}^{\prime}, a_{-i}, \alpha_{i}^{\prime}, \delta_{i}\right)-u_{i}\left(a_{i}^{\prime} \wedge a_{i}^{\prime \prime}, a_{-i}, \alpha_{i}^{\prime}, \delta_{i}\right) \\
& \leq u_{i}\left(a_{i}^{\prime} \vee a_{i}^{\prime \prime}, a_{-i}, \alpha_{i}^{\prime}, \delta_{i}\right)-u_{i}\left(a_{i}^{\prime \prime}, a_{-i}, \alpha_{i}^{\prime}, \delta_{i}\right) \\
& <u_{i}\left(a_{i}^{\prime} \vee a_{i}^{\prime \prime}, a_{-i}, \alpha_{i}^{\prime \prime}, \delta_{i}\right)-u_{i}\left(a_{i}^{\prime \prime}, a_{-i}, \alpha_{i}^{\prime \prime}, \delta_{i}\right) \leq 0,
\end{aligned}
$$

which yields a contradiction.

Second, consider strictly decreasing differences of $u_{i}$ in $\left(a_{i}, \alpha_{i}\right)$, and suppose that it is not true that $a_{i}^{\prime} \unrhd a_{i}^{\prime \prime}$. Then $a_{i}^{\prime} \triangleright a_{i}^{\prime} \vee a_{i}^{\prime \prime}$ and so using the hypothesis that $u_{i}\left(a_{i}, a_{-i}, \alpha_{i}, \delta_{i}\right)$ is for any $\left(a_{-i}, \delta_{i}\right) \in A_{-i} \times[0,1]$ supermodular in $a_{i}$ and has strictly decreasing differences in $\left(a_{i}, \alpha_{i}\right)$,

$$
\begin{aligned}
0 & \leq u_{i}\left(a_{i}^{\prime \prime}, a_{-i}, \alpha_{i}^{\prime \prime}, \delta_{i}\right)-u_{i}\left(a_{i}^{\prime} \wedge a_{i}^{\prime \prime}, a_{-i}, \alpha_{i}^{\prime \prime}, \delta_{i}\right) \\
& \leq u_{i}\left(a_{i}^{\prime} \vee a_{i}^{\prime \prime}, a_{-i}, \alpha_{i}^{\prime \prime}, \delta_{i}\right)-u_{i}\left(a_{i}^{\prime \prime}, a_{-i}, \alpha_{i}^{\prime \prime}, \delta_{i}\right) \\
& <u_{i}\left(a_{i}^{\prime} \vee a_{i}^{\prime \prime}, a_{-i}, \alpha_{i}^{\prime}, \delta_{i}\right)-u_{i}\left(a_{i}^{\prime}, a_{-i}, \alpha_{i}^{\prime}, \delta_{i}\right) \leq 0,
\end{aligned}
$$

which yields a contradiction.

The first part of the proof of Lemma 7 is analogous to Topkis (1978) (see also Topkis, 1998, Theorem 2.8.4.).

\section{Proof of Proposition 7}

Let $G$ be a submodular game with negative externalities and $\boldsymbol{\delta}=\mathbf{1}$ (i.e., unit vector), and consider the greatest equilibrium under Knightian uncertainty $\overline{\mathbf{a}}(\boldsymbol{\alpha})$ when the profile of degrees of optimism is $\boldsymbol{\alpha}$. Let $\boldsymbol{\alpha}^{\prime} \geq \boldsymbol{\alpha}$. Suppose now to the contrary that the greatest equilibrium under Knightian uncertainty $\overline{\mathbf{a}}\left(\boldsymbol{\alpha}^{\prime}\right)$ is smaller or incomparable to $\overline{\mathbf{a}}(\boldsymbol{\alpha})$. In both cases there exist a player $i$ whose equilibrium actions satisfy $\bar{a}_{i}\left(\boldsymbol{\alpha}^{\prime}\right)$ is strictly smaller or incomparable to $\bar{a}_{i}(\boldsymbol{\alpha})$. Note that $\bar{a}_{i}\left(\boldsymbol{\alpha}^{\prime}\right) \in b_{i}\left(\bar{a}_{-i}\left(\boldsymbol{\alpha}^{\prime}\right), \alpha_{i}^{\prime}, 1\right)$ and $\bar{a}_{i}(\boldsymbol{\alpha}) \in b_{i}\left(\bar{a}_{-i}(\boldsymbol{\alpha}), \alpha_{i}, 1\right)$. Since $\delta_{i}=1, b_{i}$ is constant in $a_{-i}$ by Remark 2. Hence, $b_{i}\left(\bar{a}_{-i}(\boldsymbol{\alpha}), \alpha_{i}, 1\right) \neq$ $b_{i}\left(\bar{a}_{-i}\left(\boldsymbol{\alpha}^{\prime}\right), \alpha_{i}^{\prime}, 1\right)$ only if $\boldsymbol{\alpha} \neq \boldsymbol{\alpha}^{\prime}$. By Proposition $5, b_{i}\left(\bar{a}_{-i}(\boldsymbol{\alpha}), \alpha_{i}, 1\right)$ is increasing in $\alpha_{i}$, i.e., $\bar{a}_{i}\left(\boldsymbol{\alpha}^{\prime}\right) \vee$ $\bar{a}_{i}(\boldsymbol{\alpha}) \in b_{i}\left(a_{-i}\left(\boldsymbol{\alpha}^{\prime}\right), \alpha_{i}^{\prime}, 1\right)$. Let $\tilde{a}_{i}\left(\boldsymbol{\alpha}^{\prime}\right)=\bar{a}_{i}\left(\boldsymbol{\alpha}^{\prime}\right) \vee \bar{a}_{i}(\boldsymbol{\alpha})$. Then $\tilde{a}_{i}\left(\boldsymbol{\alpha}^{\prime}\right) \unrhd \bar{a}_{i}\left(\boldsymbol{\alpha}^{\prime}\right)$. We distinguish two cases: If $\tilde{a}_{i}\left(\boldsymbol{\alpha}^{\prime}\right)=\bar{a}_{i}\left(\boldsymbol{\alpha}^{\prime}\right)$, then $\bar{a}_{i}\left(\boldsymbol{\alpha}^{\prime}\right) \unrhd \bar{a}_{i}(\boldsymbol{\alpha})$, a contradiction. Otherwise, if $\tilde{a}_{i}\left(\boldsymbol{\alpha}^{\prime}\right) \triangleright \bar{a}_{i}\left(\boldsymbol{\alpha}^{\prime}\right)$, then there is a contradiction to $\bar{a}_{i}\left(\boldsymbol{\alpha}^{\prime}\right)$ being a component of the largest equilibrium under Knightian uncertainty with $\boldsymbol{\alpha}^{\prime}$ and $\boldsymbol{\delta}=\mathbf{1}$. An analogous arguments holds for positive externalities and for the least equilibrium under Knightian uncertainty.

\section{Proof of Proposition 8}

(i) and (ii): First, by assumption, a symmetric ATS exists for $G$. We denote it by $\mathbf{a}^{\circ}$. It follows from Assumption 2 that there exists a preference $t^{\circ}=\left(\delta^{\circ}, \alpha^{\circ}\right)$ with $\delta^{\circ}=1$ (and some $\alpha^{\circ}$ ) such that there is 
a symmetric EKU with $\mathbf{a}^{*}\left(\mathbf{t}^{\circ}\right)=\mathbf{a}^{\circ}$.

Second, we show that there exists a $t^{\circ}$ with an EKU of $G, \mathbf{a}^{*}\left(\mathbf{t}^{\circ}\right)=\mathbf{a}^{\circ}$ such that $t^{\circ}$ is optimistic. It is sufficient to show that for $\delta^{\circ}=1$ there exists $\alpha^{\circ} \in[0,1]$ s.t. $\alpha^{\circ} \geq \alpha^{*}$, where $\alpha^{*}=\max \left\{\alpha^{\prime} \in\right.$ $[0,1]: \mathbf{a}^{*}\left(\boldsymbol{\alpha}^{\prime},(1, \ldots, 1)\right) \in \mathcal{E}^{\text {sym }}\left(G\left(\boldsymbol{\alpha}^{\prime},(1, \ldots, 1)\right) \cap \mathcal{E}^{\text {sym }}(G)\right\}$. Suppose to the contrary that for all $\alpha$ with $\mathbf{a}^{*}(\boldsymbol{\alpha})=\mathbf{a}^{\circ}$ we have $\alpha<\alpha^{*}$. By Proposition $7, \mathbf{a}^{*}(\boldsymbol{\alpha}) \unlhd \mathbf{a}^{*}\left(\boldsymbol{\alpha}^{*}\right), \mathbf{a}^{*}(\boldsymbol{\alpha}) \in \mathcal{E}(G(\boldsymbol{\alpha},(\mathbf{1})))$. By Lemma 2 , $\mathbf{a}^{\circ} \unrhd \mathbf{a}^{*}$. Hence we must have $\mathbf{a}^{*}(\boldsymbol{\alpha})=\mathbf{a}^{*}\left(\boldsymbol{\alpha}^{*}\right)$. But then set $\alpha=\alpha^{*}$, a contradiction.

Third, we show that $t^{\circ}$ is a globally stable fESP. In particular, we show that if the EKU $\mathbf{a}^{*}\left(\mathbf{t}^{\circ}\right)$ is a symmetric ATS, then $t^{\circ}$ is a globally stable fESP in $G$. Denote by $\mathbf{t}^{\prime}:=(\overbrace{t^{\prime}, \ldots, t^{\prime}}^{m}, t^{\circ}, \ldots, t^{\circ})$ for some $m \in\{1, \ldots, n-1\}$ and $t^{\prime} \in T_{G}$. Recall that we denote by $j$ a mutant (playing $t^{\prime}$ ) and by $i$ a non-mutant (playing $t^{\circ}$ ). By the definition of ATS,

$$
\pi\left(a_{i}^{*}\left(\mathbf{t}^{\circ}\right), \aleph^{n}\left(\mathbf{a}^{*}\left(\mathbf{t}^{\circ}\right)\right)\right) \geq \pi\left(a_{j}^{*}\left(\mathbf{t}^{\prime}\right), \aleph^{n}\left(\mathbf{a}^{*}\left(\mathbf{t}^{\circ}\right)\right)\right)
$$

for all $t^{\prime} \in T_{G}$. If $a_{i}^{*}\left(\mathbf{t}^{\circ}\right) \triangleright(\triangleleft) a_{j}^{*}\left(\mathbf{t}^{\prime}\right)$ for all mutants $j$, then $\mathbf{a}^{*}\left(\mathbf{t}^{\circ}\right) \triangleright(\triangleleft) \mathbf{a}^{*}\left(\mathbf{t}^{\prime}\right)$. This implies by decreasing differences,

$$
\pi\left(a_{i}^{*}\left(\mathbf{t}^{\circ}\right), \aleph^{n}\left(\mathbf{a}^{*}\left(\mathbf{t}^{\prime}\right)\right)\right) \geq \pi\left(a_{j}^{*}\left(\mathbf{t}^{\prime}\right), \aleph^{n}\left(\mathbf{a}^{*}\left(\mathbf{t}^{\prime}\right)\right)\right) .
$$

By Remark 2 all non-mutants have constant best response selections. Therefore we can select $\mathbf{a}^{*}\left(\mathbf{t}^{\prime}\right) \in$ $\mathcal{E}^{\text {sym }}\left(G\left(\mathbf{t}^{\prime}\right)\right)$ such that $a_{i}^{*}\left(\mathbf{t}^{\circ}\right)=a_{i}^{*}\left(\mathbf{t}^{\prime}\right)$ for all non-mutants $i$. Hence,

$$
\pi\left(a_{i}^{*}\left(\mathbf{t}^{\prime}\right), \aleph^{n}\left(\mathbf{a}^{*}\left(\mathbf{t}^{\prime}\right)\right)\right) \geq \pi\left(a_{j}^{*}\left(\mathbf{t}^{\prime}\right), \aleph^{n}\left(\mathbf{a}^{*}\left(\mathbf{t}^{\prime}\right)\right)\right) .
$$

Since this holds for all $m \in\{1, \ldots, n-1\}$ and all $t^{\prime} \in T_{G}$, we have that $t^{\circ}$ is a GfESP in $G$. The case of positive externalities follows analogously.

(iii): We note that if for all players $\pi$ is strictly concave in the player's own action $a_{i}$ on $A$ for all $a_{-i}$ on $A_{-i}$, then so is $u_{i}$ since it is a sum of strictly concave functions, each term multiplied by positive scalar, and because of aggregate externalities, the worst and best-case actions of the opponents do not depend on the player's own action. Hence, for all $i \in N$, each $a_{-i}$ and each $t_{i}$, we have that $b\left(a_{-i}, t_{i}\right)$ is a singleton. By Remark 2 that $b\left(a_{-i}, \alpha_{i}\right)$ is a constant function on $A_{-i}$ for each $\alpha_{i}$. Therefore for all non-mutants $a_{i}^{*}\left(\mathbf{t}^{\circ}\right)=a_{i}^{*}\left(\mathbf{t}^{\prime}\right)$ for all $\mathbf{a}^{*}\left(\mathbf{t}^{\prime}\right) \in \mathcal{E}^{s y m}\left(G\left(\mathbf{t}^{\prime}\right)\right)$. Hence $t^{\circ}$ is a robust fESP. This completes the proof of the proposition.

\section{Proof of Proposition 9}

Lemma 8 Suppose that the strategic game $G=\langle N, A, \pi\rangle$ has positive (resp. negative) externalities, and let $\overline{\mathbf{a}}$ and $\underline{\mathbf{a}}$ be the greatest and least combination of actions in $\mathbf{A}$. If $\underline{\mathbf{a}}$ (resp. $\overline{\mathbf{a}}$ ) is a Nash equilibrium of $G$, then $\underline{a}($ resp. $\bar{a})$ is a finite population evolutionary stable strategy in $G$.

Proof of Lemma. If $G$ has positive (resp. negative) externalities, let $a:=\underline{a}$ (resp. $a:=\bar{a}$ ). Since $\mathbf{a}=(a, \ldots, a)$ is a Nash equilibrium of $G$,

$$
\pi(a, \ldots, a) \geq \pi\left(a^{\prime}, a, \ldots, a\right) \text { for all } a^{\prime} \in A .
$$

We need to show that

$$
\pi\left(a, a^{\prime}, a, \ldots, a\right) \geq \pi\left(a^{\prime}, a, \ldots, a\right) \text { for all } a^{\prime} \in A .
$$

Given both inequalities, it is sufficient to show

$$
\pi\left(a, a^{\prime}, a, \ldots, a\right) \geq \pi(a, \ldots, a) \text { for all } a^{\prime} \in A .
$$


But last inequality follows immediately from positive (resp. negative) externalities and the fact that $a:=\underline{a}(\operatorname{resp} . a:=\bar{a})$.

Proof of Proposition 9. (i) and (ii): Suppose that the game has positive (resp. negative) aggregate externalities and consider the lowest (resp. highest) symmetric profile of actions $\underline{\mathbf{a}}$ (resp. $\overline{\mathbf{a}}$ ). By Assumption 3 this profile of actions is a Nash equilibrium of the game $G$.

If $G$ has positive (resp. negative) externalities, $a:=\underline{a}$ (resp. $a:=\bar{a}$ ) is by Lemma 8 a finite population evolutionary stable strategy in $G$.

We claim that $a$ is an ATS. Since $G$ is an aggregative game, we have by definition of fESS,

$$
\pi\left(a, \aleph^{n}\left(a^{\prime}, a, \ldots, a\right)\right) \geq \pi\left(a^{\prime}, \aleph^{n}\left(a^{\prime}, a, \ldots, a\right)\right) \text { for all } a^{\prime} \in A .
$$

By increasing differences, fESS implies ATS,

$$
\pi\left(a, \aleph^{n}(a, \ldots, a)\right) \geq \pi\left(a^{\prime}, \aleph^{n}(a, \ldots, a)\right) \text { for all } a^{\prime} \in A .
$$

By Assumption 2 there exists a preference $t$ with $\delta=1$ such that a symmetric EKU satisfies $\mathbf{a}^{*}(\mathbf{t})=\mathbf{a}$. Consider the preference $t=(\delta, \alpha)$ with $\delta=1$ and $\alpha=0$. From Proposition 6 follows that a symmetric EKU with the symmetric profile of preferences $\mathbf{t}=(t, \ldots, t)$ satisfies $\mathbf{a}^{*}(\mathbf{t})=\mathbf{a}$.

Inequality (36) implies

$$
\pi\left(a_{i}^{*}(\mathbf{t}), \aleph^{n}\left(\mathbf{a}^{*}\left(t_{j}^{\prime}, t_{-j}\right)\right)\right) \geq \pi\left(a_{j}^{*}\left(t_{j}^{\prime}, t_{-j}\right), \aleph^{n}\left(\mathbf{a}^{*}\left(t_{j}^{\prime}, t_{-j}\right)\right)\right) \text { for all } t^{\prime} \in T_{G} .
$$

By Remark 2 all non-mutants have constant best response selections. Therefore we can $\operatorname{select} \mathbf{a}^{*}\left(t_{j}^{\prime}, t_{-j}\right) \in$ $\mathcal{E}^{s y m}\left(G\left(t_{j}^{\prime}, t_{-j}\right)\right)$ such that $a_{i}^{*}(\mathbf{t})=a_{i}^{*}\left(t_{j}^{\prime}, t_{-j}\right)$ for all non-mutants with $t$ and any mutant with any $t^{\prime} \in T_{G}$. Hence

$$
\pi\left(a_{i}^{*}\left(t_{j}^{\prime}, t_{-j}\right), \aleph^{n}\left(\mathbf{a}^{*}\left(t_{j}^{\prime}, t_{-j}\right)\right)\right) \geq \pi\left(a_{j}^{*}\left(t_{j}^{\prime}, t_{-j}\right), \aleph^{n}\left(\mathbf{a}^{*}\left(t_{j}^{\prime}, t_{-j}\right)\right)\right) \text { for all } t^{\prime} \in T,
$$

i.e., $t$ is a fESP in the game $G$.

(iii) We note that if $\pi$ is strictly quasi-concave in the player's own action $a_{i}$ on $A$ for all $a_{-i}$ on $A_{-i}$, then $u_{i}(1,0)=$ is strictly quasi-concave in the player's own action $a_{i}$ on $A$. Hence $b\left(a_{-i},(1,0)\right)$ is a singleton for each $a_{-i}$. By Remark 2 we have that $b\left(a_{-i},(1,0)\right)$ is a constant function on $A_{-i}$. Hence $a_{i}^{*}(\mathbf{t})=a_{i}^{*}\left(t_{j}^{\prime}, t_{-j}\right)$ for all equilibria $\mathbf{a}^{*}\left(t_{j}^{\prime}, t_{-j}\right) \in \mathcal{E}^{s y m}\left(G\left(t_{j}^{\prime}, t_{-j}\right)\right)$. This completes the proof of the proposition.

\section{References}

[1] Acemoglu, D. and M.K. Jensen (2013). Aggregate comparative statics, Games and Economic Behavior 81, 27-49.

[2] Alger, I. and J. Weibull (2019). Evolutionary models of preference formation, Annual Reviews of Economics 11, 329-354.

[3] Alós-Ferrer, C. and A.B. Ania (2005). The evolutionary stability of perfectly competitive behavior, Economic Theory 26, 497-516.

[4] Amir, R. (1996). Cournot oligopoly and the theory of supermodular games, Games and Economic Behavior 15, 132-148.

[5] Arrow, K. and L. Hurwicz (1972). An optimality criterion for decision-making under ignorance, in: Carter, C.F. and J.L. Ford (eds.), Uncertainty and expectations in economics: Essays in honour of G.L.S. Shackle, Oxford: Basil Blackwell, 1-11. 
[6] Bade, S. (2011). Ambiguous act equilibria, Games and Economic Behavior 71, 246-260.

[7] Battigalli, P., Cerreia-Vioglio, S., Maccheroni, F., and M. Marinacci (2016). A note of comparative ambiguity aversion and justifiability, Econometrica 84, 1903-1916.

[8] Battigalli, P., Cerreia-Vioglio, S., Maccheroni, F., and M. Marinacci (2015). Self-confirming equilibrium and model uncertainty, American Ecomomic Review 105, 646-677.

[9] Boone, J.L. (1986). Parental investment and elite family structure in preindustrial states: A case study of late medieval-early modern Portuguese genealogies, American Anthropologist 88, 859-878.

[10] Bulow, J.I., Geanakoplos, J.D., and Klemperer, P.D. (1985). Multimarket oligopoly: strategic substitutes and complements, Journal of Political Economy 93, 488-511.

[11] Camerer, C. and M. Weber (1992). Recent developments in modeling preferences: Uncertainty and ambiguity, Journal of Risk and Uncertainty 5, 325-370.

[12] Chateauneuf, A., Eichberger, J., and S. Grant (2007). Choice under uncertainty with the best and worst in mind: NEO-additive capacities, Journal of Economic Theory 137, 538-567.

[13] Cooper, A.C., Dunkelberg, W., and C. Woo (1988). Entrepreneur's perceived chances of success, Journal of Business Venturing 3, 97-108.

[14] Cooper, R. (1999). Coordination games, Cambridge University Press.

[15] Corchón, L. (1994). Comparative statics for aggregative games. The strong concavity case, Mathematical Social Sciences 28, 151-165.

[16] Cornes, R. and R. Hartley (2005). The geometry of aggregative games, University of Nottingham.

[17] Dekel, E., Ely, J.C., and O. Yilankaya (2007). Evolution of preferences, Review of Economic Studies $\mathbf{7 4}, 685-704$.

[18] Dominiak, A. and B.C. Schipper (2019). Common belief in Choquet rationality with an 'attitude', University of California, Davis.

[19] Dow, J. and S.R.C. Werlang (1994). Nash equilibrium under uncertainty: Breaking down backward induction, Journal of Economic Theory 64, 305-324.

[20] Dubey, P., Haimanko, O., and A. Zapechelnyuk (2006). Strategic complements and substitutes, and potential games, Games and Economic Behavior 54, 77-94.

[21] Duersch, P., Oechssler, J., and B.C. Schipper (2013). Unbeatable imitation, Games and Economic Behavior 76, 88-96.

[22] Echenique, F. (2003). The equilibrium set of two-players games with complementarities is a sublattice, Economic Theory 22, 903-905.

[23] Eichberger, J. and A. Guerdjikova (2018). Do markets prove pessimists right? International Economic Review 59, 2259-2295.

[24] Eichberger, J. and D. Kelsey (2014). Optimism and pessimism in games, International Economic Review 55, 483-505.

[25] Eichberger, J. and D. Kelsey (2002). Strategic complements, substitutes and ambiguity: The implications for public goods, Journal of Economic Theory 106, 436-466.

[26] Eichberger, J. and D. Kelsey (2000). Non-additive beliefs and strategic equilibria, Games and Economic Behavior 30, 183-215.

[27] Eichberger, J., Kelsey, D., and B.C. Schipper (2009). Ambiguity and social interaction, Oxford Economic Papers 61, 355-379. 
[28] Eichberger, J., Kelsey, D., and B.C. Schipper (2008). Granny versus game theorist: Ambiguity in experimental games, Theory and Decision 64, 333-362.

[29] Ely, J.C. and O. Yilankaya (2001). Nash equilibrium and the evolution of preferences, Journal of Economic Theory 97, 255-272.

[30] Epstein, L. G. (1997). Preference, rationalizability and equilibrium, Journal of Economic Theory 74, 1-29.

[31] Güth, W. (1995). An evolutionary approach to explaining cooperative behavior by reciprocal incentives, International Journal of Game Theory 24, 323-344.

[32] Güth, W. and M.E. Yaari (1992). Explaining reciprocal behavior in simple strategic games: An evolutionary approach, in: Witt, U. (ed.). Explaining process and change, University of Michigan Press: Ann Arbor, MI, 23-3-4.

[33] Groes, E., H. J. Jacobsen, B. Sloth and T. Tranaes (1998). Nash equilibrium with lower probabilities, Theory and Decision 44, 37-66.

[34] Haller, H. (2000). Non-additive beliefs in solvable games, Theory and Decision 49, 313-330.

[35] Heifetz, A., Shannon, C., and Y. Spiegel (2007a). What to maximize if you must?, Journal of Economic Theory 133, 31-57.

[36] Heifetz, A., Shannon, C., and Y. Spiegel (2007b). The dynamic evolution of preferences, Economic Theory 32, 251-286.

[37] Hehenkamp, B., Leininger, W., and A. Possajennikov (2004). Evolutionary equilibrium in Tullock contests: Spite and overdissipation, European Journal of Political Economy 20, 1045-1057.

[38] Huck, S., Kirchsteiger, G., and J. Oechssler (2005). Learning to like what you have - Explaining the endowment effect. Economic Journal 115, 689-702.

[39] Hughes, A.L. (1986). Reproductive success and occupational class in eighteenth-century Lancashire, England, Social Biology 33, 109-115.

[40] Hull, T.H. and V.J. Hull (1977). The relation of economic class and fertility: An analysis of some Indonesian data, Population Studies 31, 43-57.

[41] Hurwicz, L. (1951). Optimality criteria for decision making under ignorance, Cowles Commission Discussion Paper, Statistics 370.

[42] Jaffray, J.-Y. and F. Philippe (1997). On the existence of subjective upper and lower probabilities, Mathematics of Operations Research 22, 165-185.

[43] Jensen, M.K. (2005). Existence, comparative statics, and stability in games with strategic substitutes, University of Birmingham.

[44] Jungbauer, T. and K. Ritzberger (2011). Strategic games beyond expected utility, Economic Theory 48, 377-398.

[45] Klibanoff, P. (1996). Uncertainty, decision and normal form games, Northwestern University.

[46] Knight, F. (1921). Risk, uncertainty and profit, Boston: Houghton Mifflin.

[47] Koçkesen, L., Ok, E., and R. Sethi (2000a). Evolution of interdependent preferences in aggregative games, Games and Economic Behavior 31, 303-310.

[48] Koçkesen, L., Ok, E., and R. Sethi (2000b). The strategic advantage of negatively interdependent preferences: An incomplete information scenario, Journal of Economic Theory 92, 274-299.

[49] Kukushkin, N. (1994). A fixed-point theorem for decreasing mappings, Economics Letters 46, 23-26.

[50] Lo K. C. (1996). Equilibrium in beliefs under uncertainty, Journal of Economic Theory 71, 443-484. 
[51] Lo K. C. (1999). Nash equilibrium without mutual knowledge of rationality, Economic Theory 14, 621-633.

[52] Mace, R. (1996). Biased parental investment and reproductive success in Gabbra pastoralists, Behavioral Ecology and Sociobiology 38, 75-81.

[53] Marinacci, M. (2000). Ambiguous games, Games and Economic Behavior 31, 191-219.

[54] Milgrom, P. and J. Roberts (1990). Rationalizability, learning, and equilibrium in games with strategic complementarities, Econometrica 58, 1255-1277.

[55] Milgrom, P. and C. Shannon (1994). Monotone comparative statics, Econometrica 62, 157-180.

[56] Mulder, M.B. (1987). Resources and reproductive success in women with an example from the Kipsigis of Kenya, Journal of Zoology 213, 489-505.

[57] Novshek, W. (1985). On the existence of Cournot equilibrium, Review of Economic Studies 52, $85-98$.

[58] Ok, E. and F. Vega-Redondo (2001). The evolution of individualistic preferences: An incomplete information scenario, Journal of Economic Theory 97, 231-254.

[59] Possajennikov, A. (2005). Two speed evolution of strategies and preferences in symmetric games, Theory and Decision 57, 227-263.

[60] Possajennikov, A. (2003). Evolutionary foundation of aggregative-taking behavior, Economic Theory 21, 921-928.

[61] Riedel, F. and L. Sass (2014). Ellsberg games, Theory and Decision 76, 469-509.

[62] Ryan, M. J. (2002). What do uncertainty-averse decision-makers believe?, Economic Theory 20, 47-65.

[63] Samuelson, L. (2001). Introduction to the evolution of preferences, Journal of Economic Theory 97, $225-230$.

[64] Sandholm, W. (2001). Preference evolution, two-speed dynamics and rapid social change, Review of Economic Dynamics 4, 637-679.

[65] Schaffer, M.E. (1989). Are profit-maximizers the best survivors?, Journal of Economic Behavior and Organization 12, 29-45.

[66] Schaffer, M.E. (1988). Evolutionary stable strategies for a finite population and a variable contest size, Journal of Theoretical Biology 132, 469-478.

[67] Schmeidler, D. (1989). Subjective probability and expected utility with additivity, Econometrica 57, 571-587.

[68] Schipper, B.C. (2009). Imitators and optimizers in Cournot oligopoly, Journal of Economic Dynamics and Control 33, 1981-1990.

[69] Schipper, B.C. (2003). Submodularity and the evolution of Walrasian behavior, International Journal of Game Theory 32, 471-477.

[70] Seligman, M.E.P. and P. Schulman (1986). Explanatory style as a predictor of productivity and quitting among life insurance sales agents, Journal of Personality and Social Psychology 50, 832838.

[71] Taylor, S.E. and J.D. Brown (1988). Illusion and well-being: A social perspective on mental health, Psychological Bulletin 103, 193-210.

[72] Topkis, D. M. (1998). Supermodularity and complementarity, Princeton, New Jersey: Princeton University Press. 
[73] von Stackelberg, H. (1934). Marktform und Gleichgewicht, Springer Verlag.

[74] Vega-Redondo, F. (1997). The evolution of Walrasian behavior, Econometrica 65, 375-384.

[75] Vives, X. (2000). Oligopoly pricing: Old ideas and new tools, Cambridge: The MIT Press.

[76] Wakker, P. (2001). Testing and characterizing properties of nonadditive measures through violations of the Sure-Thing principle, Econometrica 69, 1039-1059.

[77] Walker, J.M., Gardner, R., and E. Ostrom (1990). Rent dissipation in a limited-access CommonPool resource: Experimental evidence, Journal of Environmental Economics and Management 19, 203-211.

[78] Wald, A. (1951). Statistical decision functions, Chelsea: New York.

[79] Zhou L. (1994). The set of Nash equilibria of a supermodular game is a complete lattice, Games and Economic Behavior 7, 295-300. 